\title{
Roughness of fuzzy soft sets and related results
}

\author{
Zhaowen $\mathrm{Li}^{1}{ }^{*}$, Tusheng $\mathrm{Xie}^{2}$ \\ ${ }^{1}$ College of Science, Guangxi University for Nationalities, Nanning, Guangxi 530006, P.R.China \\ E-mail: lizhaowen8846@126.com \\ ${ }^{2}$ College of Mathematics and Information Science, Guangxi University, Nanning, Guangxi 530004, P.R.China \\ E-mail: tushengxie@126.com
}

Received 18 March 2014

Accepted 16 October 2014

\begin{abstract}
This paper investigates roughness of fuzzy soft sets. A pair of fuzzy soft rough approximations is proposed and their properties are given. Based on fuzzy soft rough approximations, the concept of fuzzy soft rough sets is introduced. New types of fuzzy soft sets such as full, intersection complete and union complete fuzzy soft sets are defined and supported by some illustrative examples. We obtain the structure of fuzzy soft rough sets, investigate the structure of fuzzy topologies induced by fuzzy soft sets, reveal the fact that every finite fuzzy topological space is a fuzzy soft approximation space and discuss fuzzy soft rough relations. We proved that there exists a one-to-one correspondence between the set of all fuzzy soft sets and the set of all [0,1]-valued information systems, which illustrates that we can research $[0,1]$ information systems by means of fuzzy soft sets.
\end{abstract}

Keywords: Fuzzy soft sets; Fuzzy soft rough approximations; Fuzzy soft rough sets; Fuzzy topologies; Fuzzy soft rough relations; [0,1]-valued information systems; One-to-one correspondences.

\section{Introduction}

To solve complicated problems in economics, engineering, environmental science and social science, methods in classical mathematics are not always successful because of various types of uncertainties present in these problems. There are several theories: theory of probability, theory of fuzzy sets ${ }^{27}$, rough set theory ${ }^{20}$ and the interval mathematics which we can consider as mathematical tools for dealing with uncertainties. But all these theories have their own difficulties ${ }^{18}$. To overcome these kinds of difficulties, Molodtsov ${ }^{18}$ proposed a completely new approach, which is called soft set theory, for modeling uncertainty.
Recently works on soft set theory are progressing rapidly. Maji et al. ${ }^{16}$ defined several operations on soft sets and made a theoretical study on soft set theory. Aktas and Cagman ${ }^{2}$ introduced the concept of soft groups. Jun ${ }^{12}$ introduced the concept of soft BCK/BCI-algebras. Jiang et al. ${ }^{11}$ extended soft sets with description logics. Feng et al. ${ }^{7,8}$ and Ali ${ }^{1}$ investigated the relationship among soft sets, rough sets and fuzzy sets. Ge et al. ${ }^{10}$ discussed the relationship between soft sets and topological spaces.

In soft set theory, we observe that in most cases the parameters are vague words or sentences involve vague words. Considering this point, Maji et al. ${ }^{17}$ devoted the concept of fuzzy soft sets by combining soft sets with fuzzy sets. Roy et al. ${ }^{24}$ presented

*Corresponding author: Zhaowen Li 
a fuzzy soft set theoretic approach towards decision making problems. Feng et al. ${ }^{9}$ proposed an adjustable approach to (weighted) fuzzy soft set based decision making. Yang et al. ${ }^{26}$ introduced the concept of interval-valued fuzzy soft set. Jun et al. ${ }^{13}$ discussed the applications of fuzzy soft sets to the study of BCK/BCI-algebras. Tanay et al. ${ }^{25}$ investigated the topological structure of fuzzy soft sets. Li et al. ${ }^{14}$ considered L-fuzzy soft sets based on complete Boolean lattices.

Rough set theory proposed by Pawlak ${ }^{20}$ studies intelligent systems characterized by insufficient and incomplete information. The rough set philosophy is founded on the assumption that with every object in the universe, we associate some information (data, knowledge). From a practical point of view, it is better to define basic concepts of rough set theory in terms of data. In fact, information and knowledge are stored and represented in a data table in many data analysis applications. This data table containing rows labeled by objects and columns labeled by attributes is called an information system (also known as a knowledge representation system). It is well-known that Pawlak's rough set model is based on equivalence relations. The core concept of this theory is upper and lower approximation operations, which are the operations induced by an equivalent relation on the universe. They can also be seen as a closure operator and a interior operator of the topology induced by an equivalent relation on the universe.

Soft set theory, fuzzy set theory and rough set theory are all mathematical tools to deal with uncertainty. It has been found that soft sets, fuzzy sets and rough sets are closely related concepts ${ }^{2}$. Feng et al. ${ }^{7}$ provided a framework to combine fuzzy sets, rough sets and soft sets all together, which gives several interesting concepts such as rough soft sets, soft rough sets and soft rough fuzzy sets. Based on Feng's models presented in ${ }^{7}$, Meng et al. ${ }^{19}$ proposed a new soft rough set model called a soft fuzzy rough set where a fuzzy soft set is employed to granulate the universe of discourse.

As the hybrid model combining fuzzy sets, rough sets with soft sets could be exploited to extend many practical applications such as dealing with knowl- edge acquisition in information systems with fuzzy decisions (see ${ }^{6,7,8}$ ), roughening on fuzzy soft sets deserve deeply research. Notice that Meng's model is too complicated, this paper will further investigate relationships between fuzzy soft sets and rough sets where roughening on fuzzy soft sets is considered from the angle of fuzzy points based on the models presented in ${ }^{8}$.

The remaining part of this paper is organized as follows: In Section 2, we recall some basic concepts of fuzzy sets and soft sets. In Section 3, we consider a pair of fuzzy soft rough approximations and give their properties. In Section 4, we obtain the structure of fuzzy topologies induced by fuzzy soft sets and reveal the fact that every finite fuzzy topological space is a fuzzy soft approximating space. In Section 5, we introduce the concept of fuzzy soft rough sets based on fuzzy soft rough approximations and get the structure of fuzzy soft rough sets. In Section 6, we discuss fuzzy soft rough equal relations and fuzzy soft rough belonging relations. In Section 7 , we obtain two one-to-one correspondence relationships associated with fuzzy soft sets, which expounds the broad application prospect of fuzzy soft sets. Conclusion is in Section 8.

\section{Preliminaries}

In this paper, $U$ denotes initial universe, $E^{*}$ denotes the set of all possible parameters, $E$ and $E^{\prime}$ denote two nonempty subsets of $E^{*}, I$ denotes $[0,1]$ and $2^{U}$ denotes the family of all subsets of $U$.

Throughout this paper, we only consider the case where $U$ and $E^{*}$ are both nonempty finite sets, and stipulate that $\sup \emptyset=0$.

We briefly recall some basic concepts of fuzzy sets and soft sets.

\subsection{Fuzzy sets and fuzzy topologies}

Definition 2.1 $\left(^{3}\right)$ A fuzzy set $A$ in $U$ is defined by a membership function $A: U \rightarrow I$ whose membership value $A(x)$ specifies the degree to which $x$ belongs to A for each $x \in U$.

In this paper, $I^{U}$ denotes the family of all fuzzy sets in $U .1$ represents the fuzzy set which satisfies 
$\widetilde{1}(x)=1$ for each $x \in U$ and $\widetilde{0}$ represents the fuzzy set which satisfies $\widetilde{0}(x)=0$ for each $x \in U$.

If $A, B \in I^{U}$, then some fuzzy set relations and operations are given componentwise proposed by Zadeh ${ }^{27}$ as follows:

(1) $A=B \Leftrightarrow A(x)=B(x)$ for each $x \in U$.

(2) $A \subseteq B \Leftrightarrow A(x) \leqslant B(x)$ for each $x \in U$.

(3) $(A \cup B)(x)=\max \{A(x), B(x)\}$ or $A(x) \vee$ $B(x)$ for each $x \in U$.

(4) $(A \cap B)(x)=\min \{A(x), B(x)\}$ or $A(x) \wedge$ $B(x)$ for each $x \in U$.

(5) $A^{c}(x)=1-A(x)$ for each $x \in U$.

Moreover,

$\left(\bigcup_{\alpha \in \Gamma} A_{\alpha}\right)(x)=\sup \left\{A_{\alpha}(x): \alpha \in \Gamma\right\}$ or $\vee\left\{A_{\alpha}(x): \alpha \in \Gamma\right\}$

for each $x \in U$ and

$\left(\bigcap_{\alpha \in \Gamma} A_{\alpha}\right)(x)=\inf \left\{A_{\alpha}(x): \alpha \in \Gamma\right\}$ or $\wedge\left\{A_{\alpha}(x): \alpha \in \Gamma\right\}$

for each $x \in U$, where $\left\{A_{\alpha}: \alpha \in \Gamma\right\} \subseteq I^{U}$.

Obviously, $A=B \Longleftrightarrow A \subseteq B$ and $B \subseteq A$.

A fuzzy set is called a fuzzy point in $U$, if it takes the value 0 for each $y \in U$ except one, say, $x \in U$. If its value at $x$ is $\lambda(0<\lambda \leqslant 1)$, we denote this fuzzy point by $x_{\lambda}$, where the point $x$ is called its support and $\lambda$ is called its height (see ${ }^{15,21}$ ).

For a fuzzy point $x_{\lambda}$ and $A \in I^{U}$, we defined $x_{\lambda} \in A$ by $x_{\lambda} \subseteq A$.

Obviously,

$$
x_{\lambda} \in A \Longleftrightarrow \lambda \leqslant A(x) .
$$

Example 2.2 Let $U=\left\{x_{1}, x_{2}, x_{3}, x_{4}, x_{5}, x_{6}\right\}$.

(1) Put

$A\left(x_{1}\right)=0.1, A\left(x_{2}\right)=0.7, A\left(x_{3}\right)=1, A\left(x_{4}\right)=$ $0.2, A\left(x_{5}\right)=0.5, A\left(x_{6}\right)=0.9$.

Then $A$ is a fuzzy set in $X$. We denote it by

$A=\left\{\left(x_{1}, 0.1\right),\left(x_{2}, 0.7\right),\left(x_{3}, 1\right),\left(x_{4}, 0.2\right)\right.$,

(2) We have $\left.\left(x_{5}, 0.5\right),\left(x_{6}, 0.9\right)\right\}$.

$$
\begin{aligned}
A^{c}= & \left\{\left(x_{1}, 0.9\right),\left(x_{2}, 0.3\right),\left(x_{3}, 0\right),\left(x_{4}, 0.8\right),\right. \\
& \left.\left(x_{5}, 0.5\right),\left(x_{6}, 0.1\right)\right\} .
\end{aligned}
$$

(3) Put

$$
\begin{aligned}
B=\{ & \left(x_{1}, 0.4\right),\left(x_{2}, 0.2\right),\left(x_{3}, 0.8\right),\left(x_{4}, 0.3\right), \\
& \left.\left(x_{5}, 0\right),\left(x_{6}, 0.7\right)\right\} .
\end{aligned}
$$

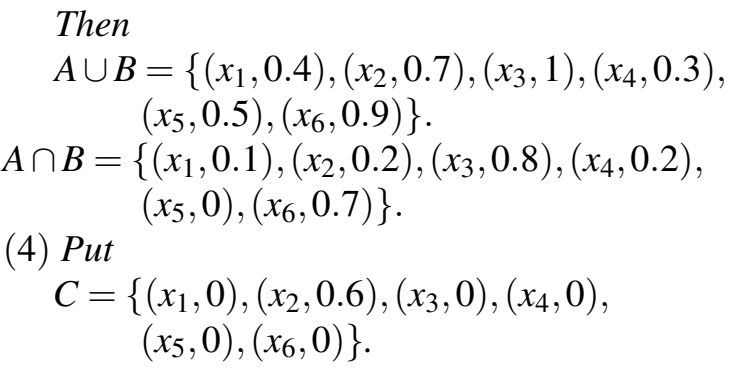

Then $C$ is a fuzzy point in $U$.

Pick $\lambda=0.6$ and $x=x_{2}$. Then $x_{\lambda}=C$.

Obviously, $x_{\lambda} \in A$ and $x_{\lambda} \notin B$.

Definition 2.3 $\left(^{3}\right)$ Let $\tau \subseteq I^{U}$. Then $\tau$ is called $a$ fuzzy topology on $U$, if

(i) $1, \widetilde{0} \in \tau$, (ii) $A, B \in \tau$ implies $A \cap B \in \tau$, (iii) $\left\{A_{\alpha}: \alpha \in \Gamma\right\} \subseteq \tau$ implies $\cup\left\{A_{\alpha}: \alpha \in \Gamma\right\} \in \tau$.

The pair $(U, \tau)$ is called a fuzzy topological space and every member of $\tau$ is called a fuzzy open set in $U . A \in I^{U}$ is called a fuzzy closed set in $U$, if $A^{c} \in \tau$.

Moreover, $\tau$ is called the indiscrete fuzzy topology on $U$, if $\tau=\{\widetilde{1}, \widetilde{0}\}$.

Let $(U, \tau)$ be a fuzzy topological space and let $A \in I^{U}$. Then interior of $A$ and the closure of $A$ (see ${ }^{3}$ ), denoted respectively by $\operatorname{int}(A)$ and $\operatorname{cl}(A)$, are defined as follows:

(1)

$$
\operatorname{int}(A)=\cup\{B: B \subseteq A \text { and } B \in \tau\},
$$

$$
c l(A)=\cap\{B: B \supseteq A \text { and } \widetilde{1}-B \in \tau\} .
$$

Definition 2.4 $\left({ }^{4,5}\right)$ Let $\tau \subseteq I^{U}$. Then $\tau$ is called a generalized fuzzy topology on $U$, if (i) $\widetilde{0} \in \tau$, (ii) $\left\{A_{\alpha}: \alpha \in \Gamma\right\} \subseteq \tau$ implies $\cup\left\{A_{\alpha}: \alpha \in \Gamma\right\} \in \tau$.

Moreover, the pair $(U, \tau)$ is called a generalized fuzzy topological space and every member of $\tau$ is called a generalized fuzzy open set in $U . A \in I^{U}$ is called a generalized fuzzy closed set in $U$, if $\widetilde{1}-A \in \tau$.

\subsection{Fuzzy soft sets}

Definition $2.5\left({ }^{18}\right)$ A pair $(f, E)$ is called a soft set over $U$, if $f$ is a mapping given by $f: E \rightarrow 2^{U}$. We denote $(f, E)$ by $f_{E}$. 
To illustrate this idea, let us consider the following typical example, which was initiated by Molodtsov ${ }^{18}$.

Example 2.6 Let $U=\left\{x_{1}, x_{2}, x_{3}, x_{4}, x_{5}, x_{6}\right\}$ be $a$ universe consisting of six houses as possible alternatives, and let $E=\left\{e_{1}, e_{2}, e_{3}, e_{4}\right\}$ be a set of parameters considered by the decision makers, where $e_{1}, e_{2}, e_{3}$ and $e_{4}$ represent the parameters "beautiful", "modern", "cheap" and "in the green surroundings", respectively.

Now, we consider a soft set $f_{E}=(f, E)$, which describes the "attractiveness of the houses" that Mr.X is going to buy. In this case, to define the soft set $f_{E}$ means to point out beautiful houses, modern houses and so on. Consider the mapping $f$ given by "houses(.)", where (.) is to be filled in by one of the parameters $e_{i} \in E$. For instance, $f\left(e_{1}\right)$ means "houses(beautiful)", and its functional value is the set consisting of all the beautiful houses in $U$. Let

$$
\begin{aligned}
& f\left(e_{1}\right)=\left\{x_{1}, x_{2}, x_{5}\right\}, f\left(e_{2}\right)=\emptyset, \\
& f\left(e_{3}\right)=\left\{x_{3}, x_{4}\right\}, f\left(e_{4}\right)=\left\{x_{3}, x_{4}, x_{6}\right\} .
\end{aligned}
$$

Then $f_{E}$ is described by the following Table 1. If $x_{i} \in f\left(e_{j}\right)$, then $x_{i j}=1$; otherwise $x_{i j}=0$, where $x_{i j}$ are the entries in the table.

Table 1. Tabular representation of the soft set $f_{E}$

\begin{tabular}{ccccccc}
\hline & $x_{1}$ & $x_{2}$ & $x_{3}$ & $x_{4}$ & $x_{5}$ & $x_{6}$ \\
\hline$e_{1}$ & 1 & 1 & 0 & 0 & 1 & 0 \\
$e_{2}$ & 0 & 0 & 0 & 0 & 0 & 0 \\
$e_{3}$ & 0 & 0 & 1 & 1 & 0 & 0 \\
$e_{4}$ & 0 & 0 & 1 & 1 & 0 & 1 \\
\hline
\end{tabular}

Definition $2.7\left({ }^{16}\right)$ A pair $(f, E)$ is called a fuzzy soft set over $U$, if $f$ is a mapping given by $f: E \rightarrow$ $I^{U}$. We also denote $(f, E)$ by $f_{E}$.

In other words, a fuzzy soft set $f_{E}$ over $U$ is a parametrized family of fuzzy sets in the universe $U$.

Example 2.8 Let $U=\left\{x_{1}, x_{2}, x_{3}, x_{4}, x_{5}, x_{6}\right\}$ and $E=$ $\left\{e_{1}, e_{2}, e_{3}\right\}$. Let $f_{E}$ be a fuzzy soft set over $U$, defined as follows

$$
\begin{aligned}
f\left(e_{1}\right)= & \left\{\left(x_{1}, 0\right),\left(x_{2}, 0.3\right),\left(x_{3}, 0.8\right),\left(x_{4}, 0.5\right),\right. \\
& \left.\left(x_{5}, 0.7\right),\left(x_{6}, 0.3\right)\right\}, \\
f\left(e_{2}\right)= & \left\{\left(x_{1}, 0.7\right),\left(x_{2}, 0.5\right),\left(x_{3}, 0.1\right),\left(x_{4}, 0.2\right),\right. \\
& \left.\left(x_{5}, 0.2\right),\left(x_{6}, 0.6\right)\right\}, \\
f\left(e_{3}\right)= & \left\{\left(x_{1}, 0.1\right),\left(x_{2}, 0.9\right),\left(x_{3}, 1\right),\left(x_{4}, 0.5\right),\right.
\end{aligned}
$$

$\left.\left(x_{5}, 0.1\right),\left(x_{6}, 0.7\right)\right\}$.

Then $f_{E}$ is described by the following Table 2.

Table 2. Tabular representation of the fuzzy soft set $f_{E}$

\begin{tabular}{ccccccc}
\hline & $x_{1}$ & $x_{2}$ & $x_{3}$ & $x_{4}$ & $x_{5}$ & $x_{6}$ \\
\hline$e_{1}$ & 0 & 0.3 & 0.8 & 0.5 & 0.7 & 0.3 \\
$e_{2}$ & 0.7 & 0.5 & 0.1 & 0.2 & 0.2 & 0.6 \\
$e_{3}$ & 0.1 & 0.9 & 1 & 0.5 & 0.1 & 0.7 \\
\hline
\end{tabular}

Definition 2.9 $\left({ }^{16}\right)$ Let $f_{E}$ and $g_{E^{\prime}}$ be two fuzzy soft sets over $U . f_{E}$ is called a fuzzy soft subset of $g_{E^{\prime}}$, if $E \subseteq E^{\prime}$ and $f(e) \subseteq g(e)$ for each $e \in E$. We denote it by $f_{E} \widetilde{\subseteq} g_{E^{\prime}}$.

Definition 2.10 $\left({ }^{16}\right)$ Let $f_{E}$ and $g_{E^{\prime}}$ be two fuzzy soft sets over $U . f_{E}$ and $g_{E^{\prime}}$ are called fuzzy soft equal, if $f_{E}$ is a fuzzy soft subset of $g_{E^{\prime}}$ and $g_{E^{\prime}}$ is a fuzzy soft subset of $f_{E}$. We denote it by $f_{E}=g_{E^{\prime}}$.

Definition 2.11 $\left({ }^{16}\right)$ Let $f_{E}$ be a fuzzy soft set over $U$.

(1) $f_{E}$ is called null, if $f(e)=\widetilde{0}$ for each $e \in E$.

(2) $f_{E}$ is called absolute, if $f(e)=\widetilde{1}$ for each $e \in E$.

Feng et al. ${ }^{8}$ introduced the concepts of full and intersection complete soft sets. This inspire us giving the following definition.

Definition 2.12 Let $f_{E}$ be a fuzzy soft set over $U$.

(1) $f_{E}$ is called full, if $\bigcup_{e \in E} f(e)=\widetilde{1}$.

(2) $f_{E}$ is called intersection complete, if for any $e_{1}, e_{2} \in E$, there exists $e_{3} \in E$ such that $f\left(e_{1}\right) \cap$ $f\left(e_{2}\right)=f\left(e_{3}\right)$.

(3) $f_{E}$ is called union complete, iffor any $e_{1}, e_{2} \in$ $E$, there exists $e_{3} \in E$ such that $f\left(e_{1}\right) \cup f\left(e_{2}\right)=$ $f\left(e_{3}\right)$.

(4) $f_{E}$ is called topological, if $\{f(e): e \in E\}$ is a fuzzy topology on $U$.

Obviously, every topological fuzzy soft set is full, intersection complete and union complete, and $f_{E}$ is intersection complete (resp. union complete) if and only if for any $E^{\prime} \subseteq E$, there exists $e^{\prime} \in E$ such that $\bigcap_{e \in E^{\prime}} f(e)=f\left(e^{\prime}\right)$ (resp. $\left.\bigcup_{e \in E^{\prime}} f(e)=f\left(e^{\prime}\right)\right)$.

Example 2.13 Let $U=\left\{x_{1}, x_{2}, x_{3}, x_{4}\right\}$ and $E=$ $\left\{e_{1}, e_{2}, e_{3}\right\}$. Let $f_{E}$ be a fuzzy soft set over $U$, de- 
fined as follows

$$
\begin{gathered}
f\left(e_{1}\right)=\left\{\left(x_{1}, 0\right),\left(x_{2}, 1\right),\left(x_{3}, 0\right),\left(x_{4}, 1\right)\right\}, f\left(e_{2}\right)=\widetilde{0}, \\
f\left(e_{3}\right)=\left\{\left(x_{1}, 1\right),\left(x_{2}, 0\right),\left(x_{3}, 1\right),\left(x_{4}, 0\right)\right\} .
\end{gathered}
$$

Obviously, $f_{E}$ is full.

We have $f\left(e_{1}\right) \cap f\left(e_{2}\right)=f\left(e_{1}\right) \cap f\left(e_{3}\right)=f\left(e_{2}\right) \cap$ $f\left(e_{3}\right)=f\left(e_{2}\right)$,

$f\left(e_{1}\right) \cup f\left(e_{3}\right)=\widetilde{1} \neq f(e)(\forall e \in E)$.

Thus $f_{E}$ is intersection complete. But $f_{E}$ is not union complete.

Example 2.14 Let $U=\left\{x_{1}, x_{2}, x_{3}, x_{4}\right\}$ and $E=$ $\left\{e_{1}, e_{2}, e_{3}\right\}$. Let $f_{E}$ be a fuzzy soft set over $U$, defined as follows

$$
\begin{gathered}
f\left(e_{1}\right)=\left\{\left(x_{1}, 0\right),\left(x_{2}, 1\right),\left(x_{3}, 0\right),\left(x_{4}, 1\right)\right\}, f\left(e_{2}\right)=\widetilde{1}, \\
f\left(e_{3}\right)=\left\{\left(x_{1}, 1\right),\left(x_{2}, 0\right),\left(x_{3}, 1\right),\left(x_{4}, 0\right)\right\} .
\end{gathered}
$$

Obviously, $f_{E}$ is full.

We have

$$
\begin{gathered}
f\left(e_{1}\right) \cup f\left(e_{2}\right)=f\left(e_{1}\right) \cup f\left(e_{3}\right)=f\left(e_{2}\right) \cup f\left(e_{3}\right)=f\left(e_{2}\right), \\
f\left(e_{1}\right) \cap f\left(e_{3}\right)=\widetilde{0} \neq f(e)(\forall e \in E) .
\end{gathered}
$$

Thus $f_{E}$ is union complete. But $f_{E}$ is not intersection complete.

Example 2.15 Let $U=\left\{x_{1}, x_{2}, x_{3}, x_{4}\right\}$ and $E=$ $\left\{e_{1}, e_{2}, e_{3}\right\}$. Let $f_{E}$ be a fuzzy soft set over $U$, defined as follows

$$
\begin{gathered}
f\left(e_{1}\right)=\left\{\left(x_{1}, 0.1\right),\left(x_{2}, 0.2\right),\left(x_{3}, 0.3\right),\left(x_{4}, 0.5\right)\right\} \\
f\left(e_{2}\right)=\left\{\left(x_{1}, 0.2\right),\left(x_{2}, 0.8\right),\left(x_{3}, 0.4\right),\left(x_{4}, 0.7\right)\right\} \\
f\left(e_{3}\right)=\left\{\left(x_{1}, 0.5\right),\left(x_{2}, 0.9\right),\left(x_{3}, 0.5\right),\left(x_{4}, 1\right)\right\}
\end{gathered}
$$

We have

$f\left(e_{1}\right) \subset f\left(e_{2}\right) \subset f\left(e_{3}\right), f\left(e_{1}\right) \cup f\left(e_{2}\right) \cup f\left(e_{3}\right)=$ $\left\{\left(x_{1}, 0.5\right),\left(x_{2}, 0.9\right),\left(x_{3}, 0.5\right),\left(x_{4}, 1\right)\right\} \neq \widetilde{1}$.

Then $f_{E}$ is intersection complete and union complete. But $f_{E}$ is not full.
From Example 2.13, 2.14 and 2.15, we have the following relationships:

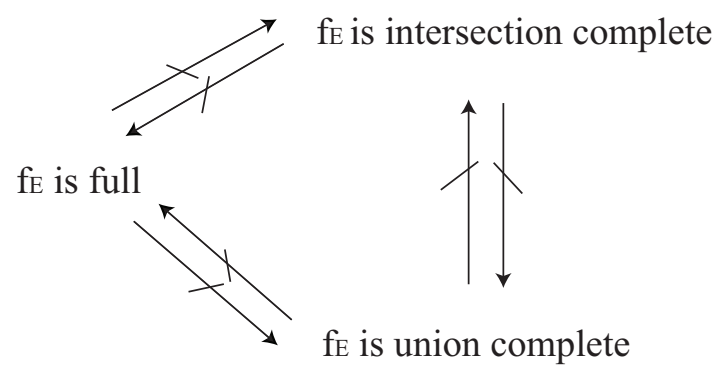

\section{Fuzzy soft rough approximations}

In ${ }^{8}$, Feng et al. introduced the concepts of soft rough sets and soft approximation spaces. Soft rough sets, which could provide a better approximation than rough sets do, can be seen as a generalized rough set model based on soft sets (see Example 4.6 in ${ }^{8}$ ). The standard soft set model is used to form the granulation structure of the universe, namely the soft approximation space. Based on this granulation structure, soft rough approximations and soft rough sets are defined.

We can follow the same research ideas when we consider fuzzy soft sets. Firstly, we introduce fuzzy soft approximation spaces. Secondly, based on the fuzzy soft approximation space $P$, we propose fuzzy soft rough approximations. Finally, we present fuzzy soft rough sets (see Definition 5.1).

In this section, we consider a pair of fuzzy soft rough approximations based on fuzzy soft approximation spaces and give their properties.

Let $f_{E}$ be a fuzzy soft set over $U, x \in U, e \in E$ and $A \in I^{U}$. Denote

$$
\begin{gathered}
\underline{A}_{x}=\left\{\lambda \in I: \exists e \in E \text { s.t. } x_{\lambda} \in f(e) \subseteq A\right\}, \\
\bar{A}_{x}=\left\{\lambda \in I: \exists e \in E \text { s.t. } x_{\lambda} \in f(e), f(e) \cap A \neq \widetilde{0}\right\} ; \\
\underline{A}_{x}^{e}=\left\{\lambda \in I: x_{\lambda} \in f(e) \subseteq A\right\}, \\
\bar{A}_{x}^{e}=\left\{\lambda \in I: x_{\lambda} \in f(e), f(e) \cap A \neq \widetilde{0}\right\} ; \\
\underline{A}^{e}(x)=\sup \underline{A}_{x}^{e}, \quad \bar{A}^{e}(x)=\sup \bar{A}_{x}^{e} .
\end{gathered}
$$


Definition 3.1 Let $f_{E}$ be a fuzzy soft set over $U$. Then the pair $P=\left(U, f_{E}\right)$ is called a fuzzy soft approximation space. Based on the fuzzy soft approximation space $P$, we define the following two operations: for $A \in I^{U}$,

$$
\begin{aligned}
& \frac{\operatorname{apr}_{P}(A)(x)=\sup \underline{A}_{x}, x \in U,}{\overline{\operatorname{apr}}_{P}(A)(x)=\sup \bar{A}_{x}, x \in U .}
\end{aligned}
$$

$\operatorname{apr}_{P}(A)$ and $\overline{a p r}_{P}(A)$ are called the fuzzy soft $P$ lower approximation and the fuzzy soft $P$-upper approximation of $U$, respectively. In general, we refer to $\operatorname{apr}_{P}(A)$ and $\overline{\operatorname{apr}}_{P}(A)$ as fuzzy soft rough approximations of $U$ with respect to $P$.

In this paper, we denote

$$
\begin{gathered}
\tau_{P}=\left\{A \in I^{U}: A=\underline{a p r}_{P}(A)\right\}, \\
\sigma_{P}=\left\{A \in I^{U}: A=\overline{a p r}_{P}(A)\right\} .
\end{gathered}
$$

Lemma 3.2 Let $f_{E}$ be a fuzzy soft set over $U, x \in U$ and $A, B \in I^{U}$. Then the following properties hold.

(1) $\underline{A}_{x} \subseteq \bar{A}_{x}$

(2) $a) \underline{A}_{x}=\bigcup_{e \in E} \underline{A}_{x}^{e}$; b) $\bar{A}_{x}=\bigcup_{e \in E} \bar{A}_{x}^{e}$.

(3) a) $\left.A \subseteq B \Rightarrow \underline{A}_{x} \subseteq \underline{B}_{x} ; \quad b\right) A \subseteq B \Rightarrow \bar{A}_{x} \subseteq \bar{B}_{x}$.

(4) If $f_{E}$ is intersection complete, then $\underline{(A \cap B)}_{x}=\underline{A}_{x} \cap \underline{B}_{x}$.

(5) ${\overline{(A \cup B)_{x}}}=\bar{A}_{x} \cup \bar{B}_{x}$.

Proof. The proofs of (1) and (2) are obvious.

(3) $a$ ) If $\underline{A}_{x}=\emptyset$. Obviously, $\underline{A}_{x} \subseteq \underline{B}_{x}$.

If $\underline{A}_{x} \neq \emptyset$. Let $\lambda \in \underline{A}_{x}$. Then $x_{\lambda} \in I^{U}$ and $x_{\lambda} \in f(e) \subseteq A$ for some $e \in E$. Since $f(e) \subseteq A$ and $A \subseteq B, f(e) \subseteq B$. This implies that $x_{\lambda} \in f(e) \subseteq B$. Thus $\lambda \in \underline{B}_{x}$.

Hence

$$
\underline{A}_{x} \subseteq \underline{B}_{x} .
$$

b) We can suppose that $\bar{A}_{x} \neq \emptyset$. Let $\lambda \in \bar{A}_{x}$. Then $x_{\lambda} \in I^{U}$ and $x_{\lambda} \in f(e), f(e) \cap A \neq \widetilde{0}$ for some $e \in E$. $f(e) \cap A \neq \widetilde{0}$ implies

$f(e)(y) \wedge A(y)=(f(e) \cap A)(y)>0$ for some $y \in U$.

Since $A \subseteq B, A(y) \leqslant B(y)$. Then

$(f(e) \cap B)(y)=f(e)(y) \wedge B(y) \geqslant f(e)(y) \wedge A(y)>0$.
Thus $f(e) \cap B \neq \widetilde{0}$. This implies that $\lambda \in \bar{B}_{x}$. Hence $\bar{A}_{x} \subseteq \bar{B}_{x}$

(4) By (3), $(A \cap B)_{x} \subseteq \underline{A}_{x} \cap \underline{B}_{x}$.

Conversely. We can suppose that $\underline{A}_{x} \cap \underline{B}_{x} \neq \emptyset$. Let $\lambda \in \underline{A}_{x} \cap \underline{B}_{x}$. Then there exist $e_{1}, e_{2} \in E$ such that

$x_{\lambda} \in I^{U}, x_{\lambda} \in f\left(e_{1}\right) \subseteq A$ and $x_{\lambda} \in f\left(e_{2}\right) \subseteq B$. Thus

$$
\lambda \leqslant f\left(e_{1}\right)(x) \text { and } \lambda \leqslant f\left(e_{2}\right)(x) .
$$

This implies that

$$
\lambda \leqslant f\left(e_{1}\right)(x) \wedge f\left(e_{2}\right)(x)=\left(f\left(e_{1}\right) \cap f\left(e_{2}\right)\right)(x)
$$

Since $f_{E}$ is intersection complete, $f\left(e_{1}\right) \cap$ $f\left(e_{2}\right)=f(e)$ for some $e \in E$. Now $\lambda \leqslant f(e)(x)$. So $x_{\lambda} \in f(e)$. Since $f\left(e_{1}\right) \subseteq A$ and $f\left(e_{2}\right) \subseteq B, f(e) \subseteq$ $A \cap B$. Thus $\lambda \in{\underline{(A \cap B)_{x}}}_{x}$ So $\underline{(A \cap B)}_{x} \supseteq \underline{A}_{x} \cap \underline{B}_{x}$.

Hence

$$
{\underline{(A \cap B)_{x}}}_{x}=\underline{A}_{x} \cap \underline{B}_{x} .
$$

(5) By (3), $\overline{(A \cup B})_{x} \supseteq \bar{A}_{x} \cup \bar{B}_{x}$.

Conversely. We can suppose that $\overline{(A \cup B})_{x} \neq \emptyset$. Let $\lambda \in \overline{(A \cup B})_{x}$. Then $x_{\lambda} \in I^{U}$ and there exists $e \in E$ such that $x_{\lambda} \in f(e), f(e) \cap(A \cup B) \neq \widetilde{0}$. Now

$$
f(e) \cap(A \cup B)=(f(e) \cap A) \cup f(e) \cap B) \neq \widetilde{0} .
$$

Thus $f(e) \cap A \neq \widetilde{0}$ or $f(e) \cap B) \neq \widetilde{0}$. This implies that $\lambda \in \bar{A}_{x} \cup \bar{B}_{x}$. So $\left.\overline{(A \cup B}\right)_{x} \subseteq \bar{A}_{x} \cup \bar{B}_{x}$.

Hence

$$
\overline{(A \cup B})_{x}=\bar{A}_{x} \cup \bar{B}_{x} .
$$

Theorem 3.3 Let $f_{E}$ be a fuzzy soft set over $U$, let $P=\left(U, f_{E}\right)$ be a fuzzy soft approximation space and $A, B \in I^{U}$. Then the following properties hold.

$$
\operatorname{apr}_{P}(A)=\bigcup_{e \in E} \underline{A}^{e}=\bigcup\{f(e): e \in E \text { and } f(e) \subseteq A\} .
$$

$\overline{\operatorname{apr}}_{P}(A)=\bigcup_{e \in E} \bar{A}^{e}=\bigcup\{f(e): e \in E$ and $f(e) \cap A \neq \widetilde{0}\}$.

(3) $\operatorname{apr}_{P}(A) \subseteq \overline{\operatorname{apr}}_{P}(A) ; \underset{\operatorname{apr}}{P}(A) \subseteq A$. 
(4) $\operatorname{apr}_{P}(\widetilde{0})=\overline{\operatorname{apr}}_{P}(\widetilde{0})=\widetilde{0} ; \quad \operatorname{apr}_{P}(\widetilde{1})=$ $\overline{\operatorname{apr}}_{P}(\widetilde{1})$.

(5) $A \subseteq B \Rightarrow \underline{a p r}_{P}(A) \subseteq \underline{a p r}_{P}(B) ; \quad A \subseteq B \Rightarrow$ $\overline{\operatorname{apr}}_{P}(A) \subseteq \overline{a p r}_{P}(B)$.

(6) $\overline{a p r}_{P}(A \cup B)=\overline{a p r}_{P}(A) \cup \overline{a p r}_{P}(B)$.

Proof. (1) and (2) can easily be obtained by decomposition of fuzzy sets.

(3) By Lemma 3.2, we can easily prove that $\underline{\operatorname{apr}}_{P}(A) \subseteq \overline{\operatorname{apr}}_{P}(A)$.

Let $x \in U$. If $\underline{A}_{x}=\emptyset$, then $\underline{a p r}_{P}(A)(x)=0 \leqslant$ $A(x)$.

If $\underline{A}_{x} \neq \emptyset$. For each $\lambda \in \underline{A}_{x}, x_{\lambda} \in I^{U}$ and $x_{\lambda} \in$ $f\left(e_{\lambda}\right) \subseteq A$ for some $e_{\lambda} \in E$. So $\lambda \leqslant f\left(e_{\lambda}\right)(x) \leqslant$ $A(x)$. This implies that $\underline{a p r}_{P}(A)(x)=\sup \underline{A}_{x} \leqslant A(x)$.

Thus

$$
\underline{\operatorname{apr}}_{P}(A) \subseteq A \text {. }
$$

(4) The proofs are obvious.

(5) Suppose that $A \subseteq B$. Let $x \in U$. We can suppose that $\underline{A}_{x} \neq \emptyset$. For each $\lambda \in \underline{A}_{x}$, by Lemma 3.2, $\lambda \in \underline{B}_{x}$. Then $\lambda \leqslant \sup \underline{B}_{x}=\underline{a p r}_{P}(B)(x)$. This implies that $\underline{\operatorname{apr}}_{P}(A)(x)=\sup \underline{A}_{x} \leqslant \underline{\operatorname{apr}}_{P}(B)(x)$, Thus

$$
\operatorname{apr}_{P}(A) \subseteq \underline{\operatorname{apr}}_{P}(B) .
$$

Similarly, we can prove that

$$
A \subseteq B \Rightarrow \overline{a p r}_{P}(A) \subseteq \overline{a p r}_{P}(B) .
$$

(6) By (5),

$$
\overline{a p r}_{P}(A \cup B) \supseteq \overline{a p r}_{P}(A) \cup \overline{a p r}_{P}(B) .
$$

Conversely. Let $x \in U$. We can suppose that $\overline{(A \cup B})_{x} \neq \emptyset$. For each $\left.\lambda \in \overline{(A \cup B}\right)_{x}, \lambda \in \bar{A}_{x} \cup \bar{B}_{x}$ by Lemma 3.2. Then $\lambda \in \bar{A}_{x}$ or $\lambda \in \bar{B}_{x}$. Thus

$\lambda \leqslant \sup \bar{A}_{x}=\overline{a p r}_{P}(A)(x)$ or $\lambda \leqslant \sup \bar{B}_{x}=\overline{\operatorname{apr}}_{P}(B)(x)$.

So

$\lambda \leqslant \overline{\operatorname{apr}}_{P}(A)(x) \vee \overline{a p r}_{P}(B)(x)=\left(\overline{a p r}_{P}(A) \cup \overline{a p r}_{P}(B)\right)(x)$.

This implies that

$$
\left.\overline{\operatorname{apr}}_{P}(A \cup B)(x)=\sup \overline{(A \cup B}\right)_{x} \leqslant\left(\overline{\operatorname{apr}}_{P}(A) \cup \overline{a p r}_{P}(B)\right)(x) .
$$

Thus

$$
\overline{a p r}_{P}(A \cup B) \subseteq \overline{a p r}_{P}(A) \cup \overline{a p r}_{P}(B) .
$$

Hence

$$
\overline{a p r}_{P}(A \cup B)=\overline{a p r}_{P}(A) \cup \overline{a p r}{ }_{P}(B) .
$$

To illustrate Theorem 3.3, we give the following examples.

Example 3.4 Let $U=\left\{x_{1}, x_{2}, x_{3}, x_{4}\right\}, E=\left\{e_{1}, e_{2}\right\}$ and let $f_{E}$ be a fuzzy soft set over $U$, defined as follows

$$
\begin{gathered}
f\left(e_{1}\right)=\left\{\left(x_{1}, 0\right),\left(x_{2}, 0.2\right),\left(x_{3}, 0.3\right),\left(x_{4}, 0\right)\right\}, \\
f\left(e_{2}\right)=\left\{\left(x_{1}, 0.2\right),\left(x_{2}, 0.8\right),\left(x_{3}, 0.1\right),\left(x_{4}, 0.7\right)\right\} .
\end{gathered}
$$

(1) Let

$$
A=\left\{\left(x_{1}, 0.5\right),\left(x_{2}, 0\right),\left(x_{3}, 0\right),\left(x_{4}, 0.7\right)\right\} .
$$

We have

$$
f\left(e_{1}\right) \cap A=\widetilde{0}, f\left(e_{2}\right) \cap A \neq \widetilde{0} .
$$

Then $\bar{A}_{x_{1}}=\bar{A}_{x_{1}}^{e_{2}}=\left\{\lambda \in(0,1]: \lambda \leqslant f\left(e_{2}\right)\left(x_{1}\right)\right\}=$ $(0,0.2]$. So

$$
\overline{\operatorname{apr}}_{P}(A)\left(x_{1}\right)=\sup \bar{A}_{x_{1}}=0.2<0.5=A\left(x_{1}\right) .
$$

Thus

$$
A \nsubseteq \overline{\operatorname{apr}}_{P}(A) \text {. }
$$

(2) Since $f\left(e_{1}\right) \subseteq \widetilde{1}, f\left(e_{2}\right) \subseteq \widetilde{1}$, $\tilde{1}_{x_{2}}=\left\{\lambda \in(0,1]: \lambda \leqslant f\left(e_{1}\right)\left(x_{2}\right)\right\} \cup\{\lambda \in(0,1]: \lambda \leqslant$ $f\left(e_{2}\right)\left(x_{2}\right)=(0,0.2] \cup(0,0.8]=(0,0.8]$.

So

$$
\operatorname{apr}_{P}(\widetilde{1})\left(x_{2}\right)=\sup \widetilde{1}_{x_{2}}=0.8<1=\widetilde{1}\left(x_{2}\right) .
$$

Thus

$$
\operatorname{apr}_{P}(\widetilde{1}) \neq \widetilde{1}
$$

(3) Let

$$
B=\left\{\left(x_{1}, 0.1\right),\left(x_{2}, 0.8\right),\left(x_{3}, 0.4\right),\left(x_{4}, 0.6\right)\right\} .
$$

Then

$A \cup B=\left\{\left(x_{1}, 0.5\right),\left(x_{2}, 0.8\right),\left(x_{3}, 0.4\right),\left(x_{4}, 0.7\right)\right\}$. 
We have

$f\left(e_{1}\right) \nsubseteq A, f\left(e_{2}\right) \nsubseteq A ; f\left(e_{1}\right) \subseteq B, f\left(e_{2}\right) \nsubseteq B ;$

$$
f\left(e_{1}\right) \subseteq A \cup B, f\left(e_{2}\right) \subseteq A \cup B .
$$

$\underline{A}_{x_{2}}=\emptyset$ implies

$$
\begin{gathered}
\underline{\operatorname{apr}}_{P}(A)\left(x_{2}\right)=0 . \\
\underline{B}_{x_{2}}=\underline{B}_{x_{2}}^{e_{1}}=\left\{\lambda \in(0,1]: \lambda \leqslant f\left(e_{1}\right)\left(x_{2}\right)\right\}=
\end{gathered}
$$
$(0,0.2]$ implies

$$
\begin{aligned}
& \underline{\operatorname{apr}}_{P}(B)\left(x_{2}\right)=\sup \underline{B}_{x_{2}}=0.2 \text {. } \\
& \underline{(A \cup B)}_{x_{2}}={\underline{(A \cup B)^{e_{1}}}}_{x_{2}}^{e_{(A)}} \underline{(A \cup B)}_{x_{2}}^{e_{2}}=\left(0, f\left(e_{1}\right)\left(x_{2}\right)\right] \cup \\
& \left(0, f\left(e_{2}\right)\left(x_{2}\right)\right]=(0,0.2] \cup(0,0.8]=(0,0.8] \text { implies } \\
& \underline{\operatorname{apr}}_{P}(A \cup B)\left(x_{2}\right)=\sup \underline{(A \cup B)}_{x_{2}}=0.8 \text {. }
\end{aligned}
$$

Note that

$\left.\underline{\operatorname{apr}}_{P}(A) \cup \underline{\operatorname{apr}}_{P}(B)\right)\left(x_{2}\right)=0.2 \neq \underline{\operatorname{apr}}_{P}(A \cup B)\left(x_{2}\right)=0.8$

Thus

$$
\underline{\operatorname{apr}}_{P}(A) \cup \underline{\operatorname{apr}}_{P}(B) \neq \underline{a p r}_{P}(A \cup B) .
$$

(4) Let

$C=\left\{\left(x_{1}, 0.2\right),\left(x_{2}, 0\right),\left(x_{3}, 0.4\right),\left(x_{4}, 0.7\right)\right\}$,

$D=\left\{\left(x_{1}, 0.4\right),\left(x_{2}, 0.6\right),\left(x_{3}, 0\right),\left(x_{4}, 0.5\right)\right\}$.

Then

$C \cap D=\left\{\left(x_{1}, 0.2\right),\left(x_{2}, 0\right),\left(x_{3}, 0\right),\left(x_{4}, 0.5\right)\right\}$.

We have

$f\left(e_{1}\right) \cap C \neq \widetilde{0}, \quad f\left(e_{2}\right) \cap C \neq \widetilde{0} ; \quad f\left(e_{1}\right) \cap D \neq$

$\widetilde{0}, f\left(e_{2}\right) \cap D \neq \widetilde{0}$;

$f\left(e_{1}\right) \cap(C \cap D)=\widetilde{0}, f\left(e_{2}\right) \cap(C \cap D) \neq \widetilde{0}$.

$\bar{C}_{x_{3}}=\bar{D}_{x_{3}}=(0,0.3]$ implies

$\overline{\operatorname{apr}}_{P}(C)\left(x_{3}\right)=\overline{\operatorname{apr}}_{P}(D)\left(x_{3}\right)=0.3$.

${\overline{(C \cap D)_{x_{3}}}}={\overline{(C \cap D)_{x_{3}}}}_{e_{2}}=(0,0.1]$ implies

Note that

$$
\overline{\operatorname{apr}}_{P}(C \cap D)\left(x_{3}\right)=0.1 \text {. }
$$

$\left(\overline{\operatorname{apr}}_{P}(C) \cap \overline{\operatorname{apr}}_{P}(D)\right)\left(x_{3}\right)=0.3 \neq \overline{\operatorname{apr}}_{P}(C \cap D)\left(x_{3}\right)=0.1$

\section{Thus}

$$
\overline{\operatorname{apr}}_{P}(C) \cap \overline{\operatorname{apr}}_{P}(D) \neq \overline{\operatorname{apr}}_{P}(C \cap D)
$$

Example 3.5 Let $U=\left\{x_{1}, x_{2}, x_{3}, x_{4}\right\}, E=\left\{e_{1}, e_{2}\right\}$ and let $f_{E}$ be a fuzzy soft set over $U$, defined as follows

$$
\begin{aligned}
& f\left(e_{1}\right)=\left\{\left(x_{1}, 0.1\right),\left(x_{2}, 0.2\right),\left(x_{3}, 0.3\right),\left(x_{4}, 0.5\right)\right\}, \\
& f\left(e_{2}\right)=\left\{\left(x_{1}, 0.2\right),\left(x_{2}, 0.8\right),\left(x_{3}, 0.1\right),\left(x_{4}, 0.7\right)\right\} . \\
& \text { Let }
\end{aligned}
$$

$A=\left\{\left(x_{1}, 0.1\right),\left(x_{2}, 0.8\right),\left(x_{3}, 0.4\right),\left(x_{4}, 0.7\right)\right\}$,

$B=\left\{\left(x_{1}, 0.3\right),\left(x_{2}, 0.8\right),\left(x_{3}, 0.1\right),\left(x_{4}, 1\right)\right\}$.

Then

$A \cap B=\left\{\left(x_{1}, 0.1\right),\left(x_{2}, 0.8\right),\left(x_{3}, 0.1\right),\left(x_{4}, 0.7\right)\right\}$.

We have

$f\left(e_{1}\right) \subseteq A, f\left(e_{2}\right) \nsubseteq A ; f\left(e_{1}\right) \nsubseteq B, f\left(e_{2}\right) \subseteq B ;$

$f\left(e_{1}\right) \nsubseteq A \cap B, f\left(e_{2}\right) \nsubseteq A \cap B$.

$\underline{A}_{x_{2}}=\underline{A}_{x_{2}}^{e_{1}}=\left\{\lambda \in(0,1]: \lambda \leqslant f\left(e_{1}\right)\left(x_{2}\right)\right\}=$ $(0,0.2]$ implies

$$
\operatorname{apr}_{P}(A)\left(x_{2}\right)=\sup \underline{A}_{x_{2}}=0.2 \text {. }
$$

$\underline{B}_{x_{2}}=\underline{B}_{x_{2}}^{e_{2}}=\left\{\lambda \in(0,1]: \lambda \leqslant f\left(e_{2}\right)\left(x_{2}\right)\right\}=$ $(0,0.8]$ implies

$$
\underline{\operatorname{apr}}_{P}(B)\left(x_{2}\right)=\sup \underline{B}_{x_{2}}=0.8 .
$$

$\underline{(A \cap B)}_{x_{2}}=\emptyset$ implies

$\operatorname{apr}_{P}(A \cap B)\left(x_{2}\right)=0$.

Note that $\left(\underline{a p r}_{P}(A) \cap \underline{a p r}_{P}(B)\right)\left(x_{2}\right)=0.2 \neq$ $\operatorname{apr}_{P}(A \cap B)\left(x_{2}\right)=0$.

Then

$\operatorname{apr}_{P}(A) \cap \underline{\operatorname{apr}}_{P}(B) \neq \underline{a p r}_{P}(A \cap B)$.

Lemma 3.6 Let $f_{E}$ be a fuzzy soft set over $U$ and $A \in I^{U}$. Then the following properties hold.

(1) If $\operatorname{apr}_{P}(A)=B$, then for each $x \in U$,

$$
\underline{A}_{x}=\underline{B}_{x} \subseteq \bar{B}_{x} \subseteq \bar{A}_{x}
$$

(2) If $\overline{\operatorname{apr}}_{P}(A)=B$, then for each $x \in U$,

$$
\underline{A}_{x} \subseteq \bar{A}_{x} \subseteq \underline{B}_{x} \subseteq \bar{B}_{x}
$$

Proof. (1) By Theorem 3.3, $\operatorname{apr}_{P}(A) \subseteq A$. Then $B \subset A$.

By Lemma 3.2, $\underline{A}_{x} \supseteq \underline{B}_{x} \subseteq \bar{B}_{x} \subseteq \bar{A}_{x}$.

It suffices to show $\underline{A}_{x} \subseteq \underline{B}_{x}$.

We can suppose that $\underline{A}_{x} \neq \emptyset$. For each $\lambda \in \underline{A}_{x}$, $x_{\lambda} \in I^{U}$ and $x_{\lambda} \in f\left(e_{\lambda}\right) \subseteq A$ for some $e_{\lambda} \in E$.

By Theorem 3.3, $B=\bigcup\{f(e): e \in E$ and $f(e) \subseteq$ $A\}$. Then $f\left(e_{\lambda}\right) \subseteq B$. This implies that $\lambda \in \underline{B}_{x}$. Thus $\underline{A}_{x} \subseteq \underline{B}_{x}$. 
(2) By Lemma 3.2,

$\underline{A}_{x} \subseteq \bar{A}_{x}$ and $\underline{B}_{x} \subseteq \bar{B}_{x}$.

It suffices to show

$$
\bar{A}_{x} \subseteq \underline{B}_{x} .
$$

We can suppose that $\bar{A}_{x} \neq \emptyset$. For each $\lambda \in \bar{A}_{x}$, $x_{\lambda} \in I^{U}$ and $x_{\lambda} \in f\left(e_{\lambda}\right), f\left(e_{\lambda}\right) \cap A \neq \widetilde{0}$ for some $e_{\lambda} \in E$.

By Theorem 3.3, $B=\bigcup\{f(e): e \in E$ and $f(e) \cap$ $A \neq \widetilde{0}\}$. Then $f\left(e_{\lambda}\right) \subseteq B$. This implies $\lambda \in \underline{B}_{x}$.

Thus $\bar{A}_{x} \subseteq \underline{B}_{x}$.

Theorem 3.7 Let $f_{E}$ be a fuzzy soft set over $U$, let $P=\left(U, f_{E}\right)$ be a fuzzy soft approximation space and $A, B \in I^{U}$. Then the following properties hold.

(1) $\operatorname{apr}_{P}\left(\underline{\operatorname{apr}}_{P}(A)\right)=\underline{\operatorname{apr}}_{P}(A)$.

(2) $\underset{\operatorname{apr}}{\operatorname{ap}}\left(\overline{\operatorname{apr}}_{P}(A)\right)=\overline{a p r}_{P}(A)$.

(3) $\overline{\operatorname{apr}}_{P}\left(\underline{\operatorname{apr}}_{P}(A)\right) \supseteq \underline{\operatorname{apr}}_{P}(A)$.

(4) $\overline{\operatorname{apr}}_{P}\left(\overline{\operatorname{apr}}_{P}(A)\right) \supseteq \overline{a p r}_{P}(A)$.

Proof. (1) By Theorem 3.3, it suffices to show that $\underline{\operatorname{apr}}_{P}(B)(x) \geqslant B(x)$ for each $x \in U$, where $B=\underline{\operatorname{apr}}_{P}(A)$.

We can suppose that $\underline{A}_{x} \neq \emptyset$. For each $\lambda \in$ $\underline{A}_{x}$, by Lemma 3.6, $\underline{A}_{x}=\underline{B}_{x}$, then $\lambda \in \underline{B}_{x}$. Thus $\lambda \leqslant \sup \underline{B}_{x}=\underline{\operatorname{apr}}_{P}(B)(x)$. Hence $B(x)=\sup \underline{A}_{x} \leqslant$ $\operatorname{apr}_{P}(B)(x)$.

(2) By Theorem 3.3, it suffices to show that $\underline{\operatorname{apr}}_{P}(B)(x) \geqslant B(x)$ for each $x \in U$, where $B=\overline{a p r}_{P}(A)$.

We can suppose that $\bar{A}_{x} \neq \emptyset$. For each $\lambda \in \bar{A}_{x}$, by Lemma 3.6, $\bar{A}_{x} \subseteq \underline{B}_{x}, \lambda \in \underline{B}_{x}$. Thus $\lambda \leqslant \sup \underline{B}_{x}=$ $\underline{\operatorname{apr}}_{P}(B)(x)$. Hence $B(x)=\sup \bar{A}_{x} \leqslant \underline{\operatorname{apr}} \underline{P}_{P}(B)(x)$.

(3) It suffices to show that

$\overline{\operatorname{apr}}_{P}(B)(x) \geqslant B(x)$ for each $x \in U$, where $B=\underline{a p r}_{P}(A)$.

We can suppose that $\underline{A}_{x} \neq \emptyset$. For each $\lambda \in \underline{A}_{x}$, by Lemma 3.6, $\underline{A}_{x}=\underline{B}_{x}, \lambda \in \underline{B}_{x}$. By Lemma 3.2, $\lambda \in \bar{B}_{x}$. Thus $\lambda \leqslant \sup \bar{B}_{x}=\overline{a p r}_{P}(B)(x)$. Hence $B(x)=\sup \underline{A}_{x} \leqslant \overline{\operatorname{apr}}_{P}(B)(x)$.

(4) It suffices to show that

$\overline{\operatorname{apr}}_{P}(B)(x) \geqslant B(x)$ for each $x \in U$, where $B=\overline{a p r}_{P}(A)$.
We can suppose that $\bar{A}_{x} \neq \emptyset$. For each $\lambda \in \bar{A}_{x}$, by Lemma 3.6, $\bar{A}_{x} \subseteq \underline{B}_{x}, \lambda \in \underline{B}_{x}$. By Lemma 3.2, $\lambda \in \bar{B}_{x}$. Thus $\lambda \leqslant \sup \bar{B}_{x}=\overline{a p r}_{P}(B)(x)$. Hence $B(x)=\sup \underline{A}_{x} \leqslant \overline{a p r}_{P}(B)(x)$.

Theorem 3.8 Let $f_{E}$ be a fuzzy soft set over $U$ and let $P=\left(U, f_{E}\right)$ be a fuzzy soft approximation space. Then the following are equivalent:

(1) $f_{E}$ is full;

(2) $A \subseteq \overline{\operatorname{apr}}_{P}(A)$ for any $A \in I^{U}$;

(3) $\overline{\operatorname{apr}}_{P}(\widetilde{1})=\widetilde{1}$;

(4) $\operatorname{apr}_{P}(\widetilde{1})=\widetilde{1}$.

Proof. $\quad(1) \Rightarrow(2)$. By Theorem 3.3, $\operatorname{apr}_{P}(A) \subseteq A$. It suffices to show that

$$
A(x) \leqslant \overline{a p r}_{P}(A)(x) \text { for each } x \in U .
$$

If $A(x)=0$, then $A(x) \leqslant \overline{a p r}_{P}(A)(x)$.

If $A(x) \neq 0$, by $f_{E}$ is full, $\widetilde{1}=\bigcup_{e \in E} f(e)$,

$$
A(x) \leqslant 1=\widetilde{1}(x)=\vee\{f(e)(x): e \in E\} .
$$

Thus $A(x) \leqslant f(e)(x)$ for some $e \in E$. Put $\lambda=$ $A(x)$. Then

$$
x_{\lambda} \in I^{U} \text { and } x_{\lambda} \in f(e) .
$$

Note that $(f(e) \cap A)(x)=f(e)(x) \wedge A(x)=A(x)$. So $\left(f(e) \cap A \neq \widetilde{0}\right.$. This implies that $\lambda \in \bar{A}_{x}$. Thus

$$
A(x)=\lambda \leqslant \overline{a p r}_{P}(A)(x) .
$$

$(2) \Rightarrow(3)$ This is obvious.

(3) $\Rightarrow$ (4) The proof follows from Theorem 3.3.

$(4) \Rightarrow(1)$. Suppose that $\underline{\operatorname{apr}}{ }_{P}(\widetilde{1})=\widetilde{1}$. By Theorem 3.3,

$$
\underline{a p r}_{P}(\widetilde{1})=\bigcup\{f(e): e \in E \text { and } f(e) \subseteq \widetilde{1}\}=\bigcup\{f(e): e \in E\} .
$$

Then $\tilde{1}=\bigcup\{f(e): e \in E\}$. Thus $f_{E}$ is full.

Theorem 3.9 Let $f_{E}$ be a fuzzy soft set over $U$ and let $P=\left(U, f_{E}\right)$ be a fuzzy soft approximation space. Then the following properties hold.

(1) If $f_{E}$ is intersection complete, then apr $_{P}(A \cap$ $B)=\operatorname{apr}_{P}(A) \cap \underline{a p r}_{P}(B)$ for any $A, B \in I^{U}$.

(2) If $f_{E}$ is full and union complete, then $\overline{\operatorname{apr}}_{P}(A)=\widetilde{1}$ for any $A \in I^{U} \backslash\{\widetilde{0}\}$. 
Proof. (1) By Theorem 3.3,

$$
\underline{a p r}_{P}(A \cap B) \subseteq \underline{a p r}_{P}(A) \cap \underline{a p r}_{P}(B) .
$$

It suffices to show that $\underline{\operatorname{apr}}_{P}(A \cap B)(x) \geqslant\left(\underline{\operatorname{apr}}_{P}(A) \cap \underline{\operatorname{apr}}_{P}(B)\right)(x)$ for each $x \in U$.

Suppose that

$\underline{\operatorname{apr}}_{P}(A \cap B)(x)<\left(\underline{\operatorname{apr}}_{P}(A) \cap \underline{\operatorname{apr}}_{P}(B)\right)(x)$ for some $x \in U$.

Put

$c=\underline{a p r}_{P}(A \cap B)(x), a=\underline{a p r}_{P}(A)(x)$ and $b=\underline{a p r}_{P}(B)(x)$.

Then $c<\min \{a, b\}$. Since $c<a$ and $a=\sup \underline{A}_{x}$, $\lambda_{1}>c$ for some $\lambda_{1} \in \underline{A}_{x}$.

Since $c<b$ and $b=\sup \underline{B}_{x}, \lambda_{2}>c$ for some $\lambda_{2} \in \underline{B}_{x}$.

$\lambda_{1} \in \underline{A}_{x}$ implies $x_{\lambda_{1}} \in I^{U}$ and $x_{\lambda_{1}} \in f\left(e_{1}\right) \subseteq A$ for some $e_{1} \in E$.

$\lambda_{2} \in \underline{B}_{x}$ implies $x_{\lambda_{2}} \in I^{U}$ and $x_{\lambda_{2}} \in f\left(e_{2}\right) \subseteq B$ for some $e_{2} \in E$.

Since $f_{E}$ is intersection complete, $f\left(e_{1}\right) \cap$ $f\left(e_{2}\right)=f(e)$ for some $e \in E$. This implies that $f(e) \subseteq A \cap B$.

Put

$$
\lambda=\min \left\{\lambda_{1}, \lambda_{1}\right\} .
$$

Then $\lambda>c$ and $x_{\lambda} \in I^{U}$. Note that $\lambda \leqslant f(e)(x)$. So $x_{\lambda} \in f(e)$. This implies that $\lambda \in \underline{(A \cap B)}_{x}$. Thus $\lambda \leqslant c$, contradiction.

(2) Since $f_{E}$ is full and union complete, $f\left(e^{*}\right)=$ $\bigcup_{e \in E} f(e)=\widetilde{1}$ for some $e^{*} \in E$. Thus, for each $A \in$ $I^{U} \backslash\{\widetilde{0}\}$ and each $x \in U$, pick $\lambda=1$, then $x_{\lambda} \in f\left(e^{*}\right)$ and $f\left(e^{*}\right) \cap A=A \neq \widetilde{0}$. So $\lambda \in \bar{A}_{x}$. This implies $\operatorname{apr}_{P}(A)(x)=1$. Hence $\overline{\operatorname{apr}}_{P}(A)=\widetilde{1}$.

Example 3.10 Let $U=\left\{x_{1}, x_{2}, x_{3}, x_{4}\right\}$ and $E=$ $\left\{e_{1}, e_{2}, e_{3}\right\}$. Let $f_{E}$ be a fuzzy soft set over $U$, defined as follows

$$
\begin{gathered}
f\left(e_{1}\right)=\left\{\left(x_{1}, 0\right),\left(x_{2}, 1\right),\left(x_{3}, 0\right),\left(x_{4}, 1\right)\right\}, f\left(e_{2}\right)=\widetilde{1}, \\
f\left(e_{3}\right)=\left\{\left(x_{1}, 1\right),\left(x_{2}, 0\right),\left(x_{3}, 1\right),\left(x_{4}, 0\right)\right\} .
\end{gathered}
$$

By Example 2.14, $f_{E}$ is full and union complete.

For any $A \in I^{U} \backslash\{\widetilde{0}\}, f\left(e_{2}\right) \cap A \neq \widetilde{0}$. By Theorem 3.3, $\overline{\operatorname{apr}}_{P}(A) \supseteq f\left(e_{2}\right)=\widetilde{1}$. Thus $\overline{\operatorname{apr}}_{P}(A)=\widetilde{1}$.

\section{Fuzzy soft sets versus fuzzy topologies}

In this section we investigate the relationship between fuzzy soft sets and fuzzy topologies.

\subsection{Fuzzy topologies induced by fuzzy soft sets}

Theorem 4.1 Let $f_{E}$ be a fuzzy soft set over $U$ and let $P=\left(U, f_{E}\right)$ be a fuzzy soft approximation space. Then the following properties hold.

(1) $\tau_{P}$ is a generalized fuzzy topology on $U$.

(2) If $f_{E}$ is full, intersection complete, then $\tau_{P}$ is a fuzzy topology on $U$.

(3) If $f_{E}$ is full and union complete, then $\sigma_{P}$ is the indiscrete fuzzy topology on $U$.

Proof. (1) By Theorem 3.3, $\widetilde{0} \in \tau_{P}$.

Let $A_{\alpha} \in \tau_{P}$ for each $\alpha \in \Gamma$. Denote $A=\cup\left\{A_{\alpha}\right.$ : $\alpha \in \Gamma\}$. Since $A_{\alpha} \subseteq A$ for each $\alpha \in \Gamma$, by Theorem 3.3, we have $A_{\alpha}=\operatorname{apr}_{P}\left(A_{\alpha}\right) \subseteq \operatorname{apr}_{P}(A)$. So $A=\cup\left\{A_{\alpha}: \alpha \in \Gamma\right\} \subseteq \operatorname{apr}_{p}(A)$. By Theorem 3.3, $\operatorname{apr}_{p}(A) \subseteq A$. Thus $\operatorname{apr}_{p}(A)=A$. This implies that $\cup\left\{A_{\alpha}: \alpha \in \Gamma\right\} \in \tau_{P}$. Hence $\tau_{P}$ is a generalized fuzzy topology on $U$.

(2) By Theorem 3.8 and 3.9, we have $\widetilde{1} \in \tau$ and $A \cap B \in \tau_{P}$ whenever $A, B \in \tau_{P}$. By (1), $\tau_{P}$ is a generalized fuzzy topology on $U$. Thus $\tau$ is a fuzzy topology on $U$.

(3) This holds by Theorem 3.3, 3.8 and 3.9.

Definition 4.2 Let $f_{E}$ be a full and intersection complete fuzzy soft set over $U$ and let $P=\left(U, f_{E}\right)$ be a fuzzy soft approximation space. Then $\tau_{P}$ is called the fuzzy topology induced by $f_{E}$ on $U$.

Example 4.3 In Example 2.13, we obtain that $\left\{A \in I^{U}: f(e) \subseteq A\right.$ for some $\left.e \in E\right\}=\left\{A_{i}: 1 \leqslant i \leqslant 8\right\}$,

where

$A_{1}=f\left(e_{1}\right)=\left\{\left(x_{1}, 0\right),\left(x_{2}, 1\right),\left(x_{3}, 0\right),\left(x_{4}, 1\right)\right\}$,

$A_{2}=f\left(e_{2}\right)=\widetilde{0}$,

$A_{3}=f\left(e_{3}\right)=\left\{\left(x_{1}, 1\right),\left(x_{2}, 0\right),\left(x_{3}, 1\right),\left(x_{4}, 0\right)\right\}$,

$A_{4}=\left\{\left(x_{1}, 1\right),\left(x_{2}, 1\right),\left(x_{3}, 0\right),\left(x_{4}, 1\right)\right\}$,

$A_{5}=\left\{\left(x_{1}, 0\right),\left(x_{2}, 1\right),\left(x_{3}, 1\right),\left(x_{4}, 1\right)\right\}$

$A_{6}=\left\{\left(x_{1}, 1\right),\left(x_{2}, 1\right),\left(x_{3}, 1\right),\left(x_{4}, 0\right)\right\}$,

$A_{7}=\left\{\left(x_{1}, 1\right),\left(x_{2}, 0\right),\left(x_{3}, 1\right),\left(x_{4}, 1\right)\right\}$,

$A_{8}=\widetilde{1}$. 
By Theorem 3.3, $\operatorname{apr}_{P}\left(A_{1}\right)=f\left(e_{1}\right) \cup f\left(e_{2}\right)=A_{1}$.

Then $A_{1} \in \tau_{P}$.

Similarly, we can prove that $A_{2}, A_{3}, A_{8} \in \tau_{P}$ and $A_{4}, A_{5}, A_{6}, A_{7} \notin \tau_{P}$.

Hence

$$
\tau_{P}=\left\{A_{1}, A_{2}, A_{3}, A_{8}\right\} .
$$

The following theorem gives the structure of fuzzy topologies induced by fuzzy soft sets.

Theorem 4.4 Let $\tau_{P}$ be the fuzzy topology induced by a full and intersection complete fuzzy soft set $f_{E}$ on $U$. Then the following properties hold.

$$
\left\{\overline{\operatorname{apr}}_{P}(A): A \in I^{U}\right\} \subseteq \tau_{P}=\left\{\underline{a p r}_{P}(A): A \in I^{U}\right\} .
$$

$$
\tau_{P} \supseteq\{f(e): e \in E\} .
$$

(3) If $f_{E}$ is topological, then

$$
\tau_{P}=\{f(e): e \in E\} .
$$

(4) $\underline{a p r}_{P}$ is an interior operator of $\tau_{P}$.

Proof. (1) By Theorem 3.7, we have

$$
\left\{\overline{\operatorname{apr}}_{P}(A): A \in I^{U}\right\} \subseteq \tau_{P} .
$$

Obviously,

$$
\tau_{P} \subseteq\left\{\operatorname{apr}_{P}(A): A \in I^{U}\right\} .
$$

Let $B \in\left\{\underline{a p r}_{P}(A): A \in I^{U}\right\}$. Then $B=$ $\operatorname{apr}_{P}(A)$ for some $A \in I^{U}$. By Theorem 3.7, $\overline{\operatorname{apr}}_{P}\left(\underline{\operatorname{apr}}_{P}(A)\right)=\underline{\operatorname{apr}}_{P}(A)$. This implies that $B \in \tau_{P}$. Thus

$$
\tau_{P} \supseteq\left\{\underline{\operatorname{apr}}_{P}(A): A \in I^{U}\right\} .
$$

Hence

$$
\left\{\overline{a p r}_{P}(A): A \in I^{U}\right\} \subseteq \tau_{P}=\left\{\underline{a p r}_{P}(A): A \in I^{U}\right\} .
$$

(2) For each $e \in E$, by Theorem 3.3,

$$
\underline{\operatorname{apr}}_{P}(f(e))=\bigcup\left\{f\left(e^{\prime}\right): e^{\prime} \in E \text { and } f\left(e^{\prime}\right) \subseteq f(e)\right\} \subseteq f(e) \text {. }
$$

Then $f(e)=\underline{a p r}_{P}(f(e))$. So $f(e) \in \tau_{P}$. Thus

$$
\{f(e): e \in E\} \subseteq \tau_{P} .
$$

(3) By (2), $\tau_{P} \supseteq\{f(e): e \in E\}$.

Let $A \in \tau_{P}$. If $A=\widetilde{0}$, by $f_{E}$ is topological, then $A \in\{f(e): e \in E\}$.

If $A \neq \widetilde{0}$, then $A=\operatorname{apr}_{P}(A)$. Put $E_{1}=\{e \in E:$ $f(e) \subseteq A\}$, by Theorem 3.3, $A=\cup\left\{f(e): e \in E_{1}\right\}$. We claim that $E_{1} \neq \emptyset$. Otherwise, $E_{1}=\emptyset$. Then for each $x \in U, A(x)=\sup \left\{f(e)(x): e \in E_{1}\right\}=$ sup $\emptyset=0$. So $A=\widetilde{0}$, a contradiction. Since $f_{E}$ is union complete, there exists $e^{\prime} \in E$ such that $\cup\left\{f(e): e \in E_{1}\right\}=f\left(e^{\prime}\right)$. That is, $A=f\left(e^{\prime}\right)$. This implies that $\tau_{P} \subseteq\{f(e): e \in E\}$.

Hence

$$
\tau_{P}=\{f(e): e \in E\} .
$$

(4) It suffices to show that

$$
\operatorname{apr}_{P}(A)=\operatorname{int}(A) \text { for each } A \in I^{U} .
$$

By (1), $\underset{\operatorname{apr}}{P}(A) \in \tau_{P} . \quad$ By Theorem 3.3, $\operatorname{apr}_{P}(A) \subseteq A$. Thus

$$
\operatorname{apr}_{P}(A) \subseteq \operatorname{int}(A) .
$$

Conversely. For each $B \in \tau_{P}$ with $B \subseteq A$, we have $B=\underline{a p r}_{P}(B) \subseteq \underline{a p r}_{P}(A)$ by Theorem 3.3. Thus

$$
\operatorname{int}(A)=\bigcup\left\{B: B \in \tau_{P} \text { and } B \subseteq A\right\} \subseteq \underline{a p r}_{P}(A) .
$$

Hence

$$
\underline{\operatorname{apr}}_{P}(A)=\operatorname{int}(A) \text {. }
$$

\subsection{Fuzzy soft sets induced by fuzzy topologies}

We recall that a fuzzy topology is finite if this fuzzy topology has only finite elements.

Definition 4.5 Let $\tau$ be a finite fuzzy topology on $U$. Denote $\tau=\left\{U_{e}: e \in E\right\}$, where $E$ is the set of indexes. Define a mapping $f_{\tau}: E \rightarrow I^{U}$ by $f_{\tau}(e)=U_{e}$ for each $e \in E$. Then the fuzzy soft set $\left(f_{\tau}\right)_{E}$ over $U$ is called the fuzzy soft set induced by $\tau$ on $U$.

Example 4.6 Let $U=\left\{x_{1}, x_{2}, x_{3}, x_{4}\right\}$ and let $\tau=$ $\left\{U_{e_{1}}, U_{e_{2}}, U_{e_{3}}, U_{e_{4}}\right\}$ be a finite fuzzy topology on $U$ where

$$
U_{e_{1}}=\left\{\left(x_{1}, 0\right),\left(x_{2}, 1\right),\left(x_{3}, 0\right),\left(x_{4}, 1\right)\right\}, U_{e_{2}}=\widetilde{0},
$$




$$
U_{e_{3}}=\left\{\left(x_{1}, 1\right),\left(x_{2}, 0\right),\left(x_{3}, 1\right),\left(x_{4}, 0\right)\right\}, U_{e_{4}}=\widetilde{1} .
$$

Now we define a mapping $f_{\tau}: E \rightarrow I^{U}$ by $f_{\tau}(e)=$ $U_{e}$ for each $e \in E=\left\{e_{1}, e_{2}, e_{3}, e_{4}\right\}$.

Thus, $\left(f_{\tau}\right)_{E}$ is the fuzzy soft set induced by $\tau$ on $U$.

Definition 4.7 Let $(U, \mu)$ be a finite fuzzy topological space. If there exists a full and intersection complete fuzzy soft set $f_{E}$ over $U$ such that $\tau_{P}=\mu$, then $(U, \mu)$ is called a fuzzy soft approximating space.

Example 4.8 Let $(U, \tau)$ be a fuzzy topological space in Example 4.6 and let $f_{E}$ be a soft set over $U$ in Example 2.13. Then $f_{E}$ is full and intersection complete.

By Example 4.3, $\tau_{P}=\tau$.

Thus $(U, \tau)$ is a fuzzy soft approximating space.

The following Proposition 4.9 can easily be proved.

Proposition 4.9 (1) Let $\tau$ be a finite fuzzy topology on $U$ and let $\left(f_{\tau}\right)_{E}$ be the fuzzy soft set induced by $\tau$ on $U$. Then $\left(f_{\tau}\right)_{E}$ is topological.

(2) Let $\tau_{1}$ and $\tau_{2}$ be two finite fuzzy topologies on $U$ and let $\left(f_{\tau_{1}}\right)_{E_{1}}$ and $\left(f_{\tau_{1}}\right)_{E_{2}}$ be two fuzzy soft sets induced respectively by $\tau_{1}$ and $\tau_{2}$ on $U$. If $\tau_{1} \subseteq \tau_{2}$, then

$$
\left(f_{\tau_{1}}\right)_{E_{1}} \widetilde{\subseteq}\left(f_{\tau_{2}}\right)_{E_{2}} .
$$

Theorem 4.10 Let $\tau$ be a finite fuzzy topology on $U$, let $\left(f_{\tau}\right)_{E}$ be the fuzzy soft set induced by $\tau$ on $U$ and let $\tau_{f_{\tau}}$ be the fuzzy topology induced by $\left(f_{\tau}\right)_{E}$ on $U$. Then $\tau=\tau_{f_{\tau}}$.

Proof. Put $\tau=\left\{U_{e}: e \in E\right\}$, then $f_{\tau}: E \rightarrow I^{U}$ is a mapping, where $f_{\tau}(e)=U_{e}$ for each $e \in E$. By Proposition 4.9, $\left(f_{\tau}\right)_{E}$ is topological.

By Theorem 4.4,

$$
\tau_{f_{\tau}}=\left\{f_{\tau}(e): e \in E\right\} .
$$

Hence

$$
\tau_{f_{\tau}}=\tau \text {. }
$$

Corollary 4.11 Every finite fuzzy topological space is a fuzzy soft approximating space.
Theorem 4.12 Let $(U, \tau)$ be a finite fuzzy topological space. Then there exists a topological fuzzy soft set $f_{E}$ over $U$ such that

$$
\operatorname{apr}_{P}(A)=\operatorname{int}(A) \text { for each } A \in I^{U},
$$

where $P=\left(U, f_{E}\right)$ is a soft approximation space.

Proof. Put $\tau=\left\{U_{e}: e \in E\right\}$, where $E$ is the set of indexes. Define a mapping $f: E \rightarrow I^{U}$ by

$$
f(e)=U_{e} \text { for each } e \in E .
$$

By Proposition $4.9, f_{E}$ is topological.

Let $A \in I^{U}$. It suffices to show that

$$
\underline{\operatorname{apr}}_{P}(A)(x)=\operatorname{int}(A)(x) \text { for each } x \in U .
$$

Let $\lambda \in \underline{A}_{x}$. Then $x_{\lambda} \in I^{U}$ and $x_{\lambda} \in f(e) \subseteq A$ for some $e \in E$. Then $\lambda \leqslant f(e)(x)=U_{e}(x)$.

Note that

$$
\operatorname{int}(A)=\bigcup\{B: B \in \tau \text { and } B \subseteq A\} .
$$

Thus

$\lambda \leqslant \bigvee\{B(x): B \in \tau$ and $B \subseteq A\}=\operatorname{int}(A)(x)$.

This implies that

$$
\operatorname{apr}_{P}(A)(x)=\sup \underline{A}_{x} \leqslant \operatorname{int}(A)(x) .
$$

On the other hand. Put

$$
C_{x}=\{B(x): B(x) \neq 0, B \in \tau \text { and } B \subseteq A\} .
$$

Let $B(x) \in C_{x}$. Then $B=f(e)$ for some $e \in E$. Pick $\lambda=B(x)$. Thus

$$
x_{\lambda} \in I^{U} \text { and } x_{\lambda} \in f(e) \subseteq A .
$$

This implies that $\lambda \in \underline{A}_{x}$. So $B(x)=\lambda \leqslant$ $\sup \underline{A}_{x}=\underline{\operatorname{apr}}_{P}(A)(x)$. Thus

$\operatorname{int}(A)(x)=\bigvee\{B(x): B \in \tau$ and $B \subseteq A\}=\sup C_{x} \leqslant \underline{a p r_{P}}(A)(x)$

Hence

$$
\operatorname{apr}_{P}(A)(x)=\operatorname{int}(A)(x) \text {. }
$$


Theorem 4.13 Let $f_{E}$ be a full and intersection complete fuzzy soft set over $U$, let $\tau_{P}$ be the fuzzy topology induced by $f_{E}$ on $U$ and let $\left(f_{\tau_{P}}\right)_{E^{\prime}}$ be the fuzzy soft set induced by $\tau_{P}$ on $U$. Then the following properties hold.

(1)

$$
f_{E} \widetilde{\simeq}\left(f_{\tau_{P}}\right)_{E^{\prime}} .
$$

(2) If $f_{E}$ is topological, then

$$
f_{E}=\left(f_{\tau_{P}}\right)_{E^{\prime}} .
$$

Proof. (1) By Theorem 4.4, $\tau_{P} \supseteq\{f(e): e \in E\}$. Denote $\tau_{P}=\left\{U_{e}: e \in E^{\prime}\right\}$, where $E \subseteq E^{\prime}$ and $U_{e}=f(e)$ for eache $e$

Thus $f_{\tau_{P}}$ is a mapping given by $f_{\tau_{P}}: E^{\prime} \rightarrow I^{U}$, where $f_{\tau_{P}}(e)=U_{e}$ for each $e \in E^{\prime}$.

Hence $f_{E} \widetilde{\subseteq}\left(f_{\tau_{P}}\right)_{E^{\prime}}$.

(2) Since $f_{E}$ is topological, By Theorem 4.4, $E=E^{\prime}$.

Hence $f_{E}=\left(f_{\tau_{P}}\right)_{E^{\prime}}$.

\section{The related properties of fuzzy soft rough sets}

In this section we introduce the concept of fuzzy soft rough sets and give the related properties of fuzzy soft rough sets.

Definition 5.1 Let $f_{E}$ be a fuzzy soft set over $U$ and let $P=\left(U, f_{E}\right)$ be a fuzzy soft approximation space. Then for each $A \in I^{U}, A$ is called a fuzzy soft $P$ definable set, if $\underline{a p r}_{P}(A)=\overline{a p r}_{P}(A) ; A$ is called a fuzzy soft $P$-rough set, if $\operatorname{apr}_{P}(A) \neq \overline{\operatorname{apr}}_{P}(A)$.

Moreover, the sets

$$
\begin{gathered}
\operatorname{Pos}_{P}(A)=\underline{a p r}_{P}(A), \\
\operatorname{Neg}_{P}(A)=\widetilde{1}-\overline{a p r}_{P}(A), \\
\operatorname{Bnd}_{P}(A)=\overline{a p r}_{P}(A)-\underline{a p r}_{P}(A)
\end{gathered}
$$

are called the soft P-positive region, the soft $P$ negative region and the soft $P$-boundary region of A, respectively.
Example 5.2 Let $U=\left\{x_{1}, x_{2}, x_{3}, x_{4}\right\}$ and $E=$ $\left\{e_{1}, e_{2}, e_{3}\right\}$. Let $f_{E}$ be a fuzzy soft set over $U$, defined as follows

$$
\begin{aligned}
f\left(e_{1}\right) & =\left\{\left(x_{1}, 0.3\right),\left(x_{2}, 0\right),\left(x_{3}, 0\right),\left(x_{4}, 0.4\right)\right\}, \\
f\left(e_{2}\right) & =\left\{\left(x_{1}, 0.2\right),\left(x_{2}, 0.4\right),\left(x_{3}, 0.6\right),\left(x_{4}, 0.3\right)\right\}, \\
f\left(e_{3}\right) & =\left\{\left(x_{1}, 0.9\right),\left(x_{2}, 0.2\right),\left(x_{3}, 0.5\right),\left(x_{4}, 0.3\right)\right\} . \\
\text { Let } &
\end{aligned}
$$

$$
A=\left\{\left(x_{1}, 0.4\right),\left(x_{2}, 0.1\right),\left(x_{3}, 0\right),\left(x_{4}, 0.7\right)\right\} .
$$

Since $f\left(e_{1}\right) \widetilde{\subset} A, f\left(e_{2}\right) \widetilde{\not \subset} A$ and $f\left(e_{3}\right) \widetilde{\not \subset} A$, by Theorem 3.3, we have apr ${ }_{P}(A)=f\left(e_{1}\right)$.

$\in$ E. Since $f\left(e_{i}\right) \cap A \neq \widetilde{0}(i=1,2,3)$, by Theorem 3.3, we have $\overline{\operatorname{apr}}_{P}(A)=f\left(e_{1}\right) \cup f\left(e_{2}\right) \cup f\left(e_{3}\right)$.

Thus $\operatorname{apr}_{P}(A) \neq \overline{\operatorname{apr}}_{P}(A)$. This show that $A$ is a fuzzy soft rough set.

Denote \},

$$
\mathscr{R}=\left\{A \in I^{U}: A \text { is a fuzzy soft } P\right. \text {-rough set }
$$

set \}.

$$
\mathscr{D}=\left\{A \in I^{U}: A \text { is a fuzzy soft } P\right. \text {-definable }
$$

Theorem 5.3 Let $f_{E}$ be a fuzzy soft set over $U$ and let $P=\left(U, f_{E}\right)$ be a fuzzy soft approximation space. Then for each $A \in I^{U}$,

$$
A \in \mathscr{D} \Longleftrightarrow \overline{\operatorname{apr}}_{P}(A) \subseteq A .
$$

Proof. Note that if $A$ is fuzzy soft $P$-definable, then $\operatorname{apr}_{P}(A)=\overline{a p r}_{P}(A)$. By Theorem 3.3, $\underline{\operatorname{apr}}_{P}(A) \subseteq A$. Thus $\overline{\operatorname{apr}}_{P}(A) \subseteq A$.

Conversely. Suppose that $\overline{a p r}_{P}(A) \subseteq A$. To prove that $A$ is fuzzy soft $P$-definable, we only need to show that

$$
\overline{\operatorname{apr}}_{P}(A)(x)=\underline{\operatorname{apr}}_{P}(A)(x) \text { for each } x \in U .
$$

Put $B=\overline{a p r}_{P}(A)$. By Lemma 3.6, $\bar{A}_{x} \subseteq \underline{B}_{x}$. Since $B \subseteq A$, by Lemma 3.2, we have $\underline{B}_{x} \subseteq \underline{A}_{x}$. This implies that $\bar{A}_{x}=\underline{A}_{x}$. Hence

$$
\overline{\operatorname{apr}}_{P}(A)(x)=\sup \bar{A}_{x}=\sup \underline{A}_{x}=\underline{a p r}_{P}(A)(x) .
$$


Corollary 5.4 Let $f_{E}$ be a fuzzy soft set over $U$, let $P=\left(U, f_{E}\right)$ be a fuzzy soft approximation space. Then for each $A \in I^{U}$,

$$
A \in \mathscr{R} \Longleftrightarrow \overline{\operatorname{apr}}_{P}(A) \nsubseteq A .
$$

For $\mathscr{P}, \mathscr{Q} \subseteq I^{U}$, denote

$$
\mathscr{P} \backslash \mathscr{Q}=\left\{A \in I^{U}: A \in \mathscr{P} \text { and } A \notin \mathscr{Q}\right\} .
$$

The following Theorem 5.6 gives the structure of fuzzy soft rough sets.

Theorem 5.5 Let $f_{E}$ be a fuzzy soft set over $U$ and let $P=\left(U, f_{E}\right)$ be a soft approximation space.

(1) $\mathscr{R} \cup \mathscr{D}=I^{U}, \mathscr{R} \cap \mathscr{D}=\emptyset$ and $\sigma_{P} \subseteq \mathscr{D}$.

(2) If $f_{E}$ is full, then

$$
\mathscr{R}=I^{U} \backslash \sigma_{P} \text { and } \mathscr{D}=\sigma_{P} \subseteq \tau_{P} .
$$

(2) If $f_{E}$ is full and union complete, then

$$
\mathscr{R}=I^{U} \backslash\{\widetilde{0}, \widetilde{1}\} \text { and } \mathscr{D}=\{\widetilde{0}, \widetilde{1}\}=\sigma_{P} \subseteq \tau_{P} .
$$

Proof. This holds by Theorem 3.3, 3.8, 3.9 and 5.3. 5.6.

By Theorem 3.9, we have the following Theorem

Theorem 5.6 Let $f_{E}$ be a full and union complete fuzzy soft set over $U$. Then for $A \in I^{U} \backslash\{\widetilde{0}\}$,

$$
\operatorname{Neg}_{P}(A)=\widetilde{0}, \operatorname{Bnd}_{P}(A)=\widetilde{1}-\operatorname{Pos}_{P}(A),
$$

where $P=\left(U, f_{E}\right)$ is a fuzzy soft approximation space.

\section{Fuzzy soft rough relations}

In this section we discuss fuzzy soft rough equal relations and fuzzy soft rough belonging relations.

\subsection{Fuzzy soft rough equal relations}

Definition 6.1 Let $f_{E}$ be a fuzzy soft set over $U$ and let $P=\left(U, f_{E}\right)$ be a fuzzy soft approximation space. For $A, B \in I^{U}$, we define

$$
A \bar{\sim}_{P} B \Longleftrightarrow \overline{a p r}_{P}(A)=\overline{a p r}_{P}(B),
$$

and

$$
A \simeq_{P} B \Longleftrightarrow \underline{a p r}_{P}(A)=\underline{a p r}_{P}(B) .
$$

These binary relations are called the upper fuzzy soft rough equal relation and the lower fuzzy soft rough equal relation, respectively.

It is easy to verify that the relations defined above are all equivalence relations on $I^{U}$.

Proposition 6.2 Let $f_{E}$ be a fuzzy soft set over $U$ and let $P=\left(U, f_{E}\right)$ be a fuzzy soft approximation space. Let $A, A^{\prime}, B, B^{\prime} \in I^{U}$. Then the following properties hold.

(1) $A \widetilde{\sim}_{P} B \Longleftrightarrow A \cup B \bar{\sim}_{P} A$ and $A \cup B \bar{\sim}_{P} B$;

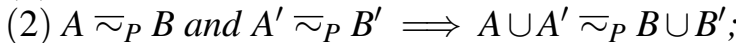

(3) $A \bar{\sim}_{P} B \Longrightarrow A \cup(\widetilde{1}-B) \bar{\sim}_{P} \widetilde{1}$;

(4) $A \subseteq B$ and $B \widetilde{\sim}_{P} \widetilde{0} \Longrightarrow A \widetilde{\sim}_{P} \widetilde{0}$;

(5) $A \subseteq B$ and $A \widetilde{\sim}_{P} \widetilde{1} \Longrightarrow B \bar{\sim}_{P} \widetilde{1}$.

(6) If $f_{E}$ is full and union complete, then $A \widetilde{\sim}_{P} B$ for any $A, B \in 2^{U} \backslash\{\widetilde{0}\}$.

Proof. (1) If $A \bar{\sim}_{P} B$, then $\overline{a p r}_{P}(A)=$ $\overline{a p r}_{P}(B) . \quad$ By Theorem 3.3, $\overline{a p r}_{P}(A \cup B)=$ $\overline{a p r}_{P}(A) \cup \overline{a p r}_{P}(B)$. This implies that $\overline{a p r}_{P}(A \cup B)=$ $\overline{a p r}_{P}(A)=\overline{a p r}_{P}(B)$. Thus $A \cup B \widetilde{\sim}_{P} A$ and $A \cup$ $B \bar{\sim}_{P} B$

Conversely. If $A \cup B \widetilde{\sim}_{P} A$ and $A \cup B \bar{\sim}_{P} B$, then $A \widetilde{\sim}_{P} B$ because $\bar{\sim}_{P}$ is a equivalence relation.

(2) Let $A \bar{\sim}_{P} B$ and $A^{\prime} \bar{\sim}_{P} B^{\prime}$. Then $\overline{a p r}_{P}(A)=$ $\overline{a p r}_{P}(B), \overline{a p r}_{P}\left(A^{\prime}\right)=\overline{a p r}_{P}\left(B^{\prime}\right)$. By Theorem 3.3, $\overline{a p r}_{P}\left(A \cup A^{\prime}\right)=\overline{a p r}_{P}(A) \cup \overline{a p r}_{P}\left(A^{\prime}\right), \overline{a p r}_{P}\left(B \cup B^{\prime}\right)=$ $\overline{a p r}_{P}(B) \cup \overline{a p r}_{P}\left(B^{\prime}\right)$. This implies that $\overline{\operatorname{apr}}_{P}(A \cup$ $\left.A^{\prime}\right)=\overline{a p r}_{P}\left(B \cup B^{\prime}\right)$. Thus $A \cup A^{\prime} \bar{\sim}_{P} B \cup B^{\prime}$.

(3) Suppose that $A \bar{\sim}_{P} B$. Then $\overline{a p r}_{P}(A)=$ $\overline{a p r}_{P}(B)$. By Theorem 3.3, $\overline{a p r}_{P}(A \cup(\widetilde{1}-B))=$ $\overline{a p r}_{P}(A) \cup \overline{a p r}_{P}(\widetilde{1}-B)$. This implies that $\overline{a p r}_{P}(A \cup$ $(\widetilde{1}-B))=\overline{a p r}_{P}(B) \cup \overline{a p r}_{P}(\widetilde{1}-B)=\overline{a p r}_{P}(\widetilde{1})$. Thus $A \cup(\widetilde{1}-B) \widetilde{\sim}_{P} \widetilde{1}$

(4) Assume $A \subseteq B$ and $B \widetilde{\sim}_{P} \widetilde{0}$. By Theorem 3.3, $\overline{a p r}_{P}(A) \subseteq \overline{a p r}_{P}(B)=\overline{a p r}_{P}(\widetilde{0})=\widetilde{0}$. Then $\overline{a p r}_{P}(A)=\widetilde{0}=\overline{a p r}_{P}(\widetilde{0})$. Thus $A \widetilde{\sim}_{P} \widetilde{0}$.

(5) Suppose that $A \subseteq B$ and $A \widetilde{\sim}_{P} \widetilde{1}$. By Theorem 3.3, $\overline{\operatorname{apr}}_{P}(B) \supseteq \overline{\operatorname{apr}}_{P}(A)=\overline{a p r}_{P}(\widetilde{1})$. Since $B \subseteq \widetilde{1}$, $\overline{a p r}_{P}(B) \subseteq \overline{\operatorname{apr}}_{P}(\widetilde{1})$. This implies that $\overline{\operatorname{apr}}_{P}(B)=$ $\overline{\operatorname{apr}}_{P}(\widetilde{1})$. Thus $B \bar{\sim}_{P} \widetilde{1}$.

(6) This holds by Theorem 3.9. 
By Theorem 3.3 and 3.9, we can prove the following Proposition 6.3.

Proposition 6.3 Let $f_{E}$ be a fuzzy soft set over $U$ and let $P=\left(U, f_{E}\right)$ be a fuzzy soft approximation space. Let $A, A^{\prime}, B, B^{\prime} \in 2^{U}$. Then the following properties hold.

(1) If $f_{E}$ is intersection complete, then $A \simeq_{P} B \Longleftrightarrow A \cap B \simeq_{P} A$ and $A \cap B \simeq_{P} B ;$

(2) If $f_{E}$ is intersection complete, then $A \simeq_{P} B$ and $A^{\prime} \simeq_{P} B^{\prime} \Longrightarrow A \cap A^{\prime} \simeq_{P} B \cap B^{\prime} ;$

(3) $A \subseteq B$ and $B \simeq_{P} \widetilde{0} \Longrightarrow A \simeq_{P} \widetilde{0}$;

(4) $A \subseteq B$ and $A \simeq_{P} \widetilde{1} \Longrightarrow B \simeq_{P} \widetilde{1}$.

Theorem 6.4 Let $f_{E}$ be a fuzzy soft set over $U$ and let $P=\left(U, f_{E}\right)$ be a fuzzy soft approximation space. Let $A, B \in I^{U}$. Then the following properties hold.

$$
\operatorname{apr}_{P}(A)=\cap\left\{B \in I^{U}: A \simeq_{P} B\right\} .
$$

(2) If $f_{E}$ is full, then

$$
\overline{\operatorname{apr}}_{P}(A) \supseteq \cup\left\{B \in I^{U}: A \bar{\sim}_{P} B\right\} .
$$

Proof. (1) For any $B \in\left\{B \in I^{U}: A \simeq_{P} B\right\}$, $A \simeq_{P} B$, then $\operatorname{apr}_{P}(A)=\underset{\operatorname{apr}}{P}(B)$. By Theorem 3.3, $\operatorname{apr}_{P}(B) \subseteq B$. This implies that $\operatorname{apr}_{P}(A) \subseteq B$. Thus

$$
\underline{\operatorname{apr}}_{P}(A) \subseteq \cap\left\{B \in I^{U}: A \simeq_{P} B\right\} .
$$

Conversely. By Theorem 3.7, $\operatorname{apr}_{P}\left(\underline{\operatorname{apr}}_{P}(A)\right)=$ $\operatorname{apr}_{P}(A)$. That is, $A \simeq_{P} \underline{\operatorname{apr}}_{P}(A)$. Then $\underline{\operatorname{apr}}_{P}(A) \in$ $\left\{B \in I^{U}: A \simeq_{P} B\right\}$. Thus

$$
\operatorname{apr}_{P}(A) \supseteq \cap\left\{B \in I^{U}: A \simeq_{P} B\right\} .
$$

Hence

$$
\underline{\operatorname{apr}}_{P}(A)=\cap\left\{B \in I^{U}: A \simeq_{P} B\right\} .
$$

(2) For any $B \in\left\{B \in I^{U}: A \bar{\sim}_{P} B\right\}, A{\sim_{P}} B$, then $\overline{a p r}_{P}(A)=\overline{a p r}_{P}(B)$. Since $f_{E}$ is full, by Theorem 3.8, we have $B \subseteq \overline{a p r}_{P}(B)$. This implies that $B \subseteq \overline{\operatorname{apr}}_{P}(A)$. Thus

$$
\overline{\operatorname{apr}}_{P}(A) \supseteq \cup\left\{B \in I^{U}: A \bar{\sim}_{P} B\right\} .
$$

\subsection{Fuzzy soft rough belonging relations}

Definition 6.5 Let $f_{E}$ be a fuzzy soft set over $U$ and let $P=\left(U, f_{E}\right)$ be a fuzzy soft approximation space. For any $x_{\lambda} \in P(U)$ and $A \in I^{U}$, we define

$x_{\lambda} \underline{\in}_{P} A \Longleftrightarrow x_{\lambda} \in \operatorname{apr}_{P}(A)$ and $x_{\lambda} \bar{\epsilon}_{P} A \Longleftrightarrow$ $x_{\lambda} \in \overline{\operatorname{apr}}_{P}(A)$,

where $P(U)$ is the set of all fuzzy points in $U$.

These binary relations are called the lower fuzzy soft rough belonging relation and the upper fuzzy soft rough belonging relation, respectively.

Example 6.6 Let $U=\left\{x_{1}, x_{2}, x_{3}, x_{4}\right\}, E=\left\{e_{1}, e_{2}\right\}$ and let $f_{E}$ be a fuzzy soft set over $U$, defined as follows

$$
\begin{aligned}
& f\left(e_{1}\right)=\left\{\left(x_{1}, 0.4\right),\left(x_{2}, 0.3\right),\left(x_{3}, 0\right),\left(x_{4}, 0.6\right)\right\} \\
& f\left(e_{2}\right)=\left\{\left(x_{1}, 0.2\right),\left(x_{2}, 0.8\right),\left(x_{3}, 0.1\right),\left(x_{4}, 0.7\right)\right\} \\
& \text { Let }
\end{aligned}
$$

$$
A=\left\{\left(x_{1}, 0.5\right),\left(x_{2}, 0.6\right),\left(x_{3}, 0\right),\left(x_{4}, 0.7\right)\right\} .
$$

Then $\left(x_{2}\right)_{0.2} \underline{\epsilon}_{P} A$ and $\left(x_{2}\right)_{0.7} \bar{\epsilon}_{P} A$

By Theorem 3.3, 3.7, 3.8 and 3.9, we can prove the following three Propositions.

Proposition 6.7 Let $f_{E}$ be a fuzzy soft set over $U$ and let $P=\left(U, f_{E}\right)$ be a fuzzy soft approximation space. Let $A, A^{\prime}, B, B^{\prime} \in 2^{U}$. Then the following properties hold.

(1) $x_{\lambda} \in_{P} A \Longleftrightarrow$ there exists $e \in E$ such that $x_{\lambda} \in f(e) \subseteq A$.

(2) $x_{\lambda} \underline{\in}_{P} A \Longrightarrow x_{\lambda} \in A$.

(3) $x_{\lambda} \in_{P} A$ and $A \subseteq B \Longrightarrow x_{\lambda} \in_{P} B$.

(4) $x_{\lambda} \in_{P} A \Longleftrightarrow x_{\lambda} \in_{P} \operatorname{apr}_{P}(A)$.

(5) If $f_{E}$ is intersection complete, then $x_{\lambda} \in_{P} A \cap$ $B \Longleftrightarrow x_{\lambda} \underline{\epsilon}_{P} A$ and $x_{\lambda} \underline{\in}_{P} B$.

Proposition 6.8 Let $f_{E}$ be a fuzzy soft set over $U$ and let $P=\left(U, f_{E}\right)$ be a fuzzy soft approximation space. Let $A, A^{\prime}, B, B^{\prime} \in 2^{U}$. Then the following properties hold.

(1) $x_{\lambda} \bar{\in}_{P} A \Longleftrightarrow$ there exists $e \in E$ such that $x_{\lambda} \in f(e)$ and $f(e) \cap A \neq \widetilde{0}$.

(2) If $f_{E}$ is full, then $x_{\lambda} \in A \Longrightarrow x_{\lambda} \bar{\epsilon}_{P} A$.

(3) $x_{\lambda} \bar{\epsilon}_{P} A$ and $A \subseteq B \Longrightarrow x_{\lambda} \bar{\epsilon}_{P} B$.

(4) $x_{\lambda} \bar{\epsilon}_{P} A \cup B \Longleftrightarrow x_{\lambda} \bar{\epsilon}_{P} A$ or $x_{\lambda} \bar{\epsilon}_{P} B$. 
Proposition 6.9 Let $f_{E}$ be a fuzzy soft set over $U$ and let $P=\left(U, f_{E}\right)$ be a fuzzy soft approximation space. Let $A, A^{\prime}, B, B^{\prime} \in 2^{U}$. Then the following properties hold.
(1) $x_{\lambda} \underline{\in}_{P} A \Longrightarrow x_{\lambda} \bar{\epsilon}_{P} A$.
(2) $x_{\lambda} \bar{\epsilon}_{P} A \Longleftrightarrow x_{\lambda} \in_{P} \overline{\operatorname{apr}}_{P}(A)$.
(3) $x_{\lambda} \in_{P} A \Longrightarrow x_{\lambda} \bar{\in}_{P} \operatorname{apr}_{P}(A)$.
(4) $x_{\lambda} \bar{\epsilon}_{P} A \Longrightarrow x_{\lambda} \bar{\epsilon}_{P} \overline{\overline{a p r}}_{P}(A)$.

\section{Some correspondence relationships associated with fuzzy soft sets}

Feng et al. ${ }^{7}$ researched the relationships among soft sets, fuzzy sets and rough sets, and obtained some good results. Inspired by their work, we investigate some correspondence relationships associated with fuzzy soft sets in this section.

Definition 7.1 Let $f_{E}$ be a fuzzy soft set over U. Define a fuzzy relation $R_{f}$ from $E$ to $U$ by

$$
R_{f}(e, x)=f(e)(x)
$$

for each $(e, x) \in E \times U$. Then $R_{f}$ is called the fuzzy relation from $E$ to $U$ induced by $f_{E}$.

Definition 7.2 Let $R$ be a fuzzy relation from $E$ to $U$. Define a mapping $f_{R}: E \rightarrow I^{U}$ by

$$
f_{R}(e)(x)=R(e, x)(x \in U)
$$

for each $e \in E$. Then $\left(f_{R}\right)_{E}$ is called the fuzzy soft set induced by $R$.

Lemma 7.3 Let $R$ be a fuzzy relation from $E$ to $U$, let $\left(f_{R}\right)_{E}$ be the fuzzy soft set induced by $R$ and let $R_{f_{R}}$ be the fuzzy relation from $E$ to $U$ induced by $\left(f_{R}\right)_{E}$. Then $R=R_{f_{R}}$.

Proof. For each $(e, x) \in E \times U$,

$$
R_{f_{R}}(e, x)=f_{R}(e)(x) .
$$

Note that $f_{R}(e)(x)=R(e, x)$. Then $R_{f_{R}}(e, x)=$ $R(e, x)$.

Thus

$$
R=R_{f_{R}}
$$

Lemma 7.4 Let $f_{E}$ be a fuzzy soft set over $U$, let $R_{f}$ be the fuzzy relation from $E$ to $U$ induced by $f_{E}$ and let $\left(f_{R_{f}}\right)_{E}$ be the fuzzy soft set induced by $R_{f}$. Then

$$
f_{E}=\left(f_{R_{f}}\right)_{E} .
$$

Proof. Since $f_{R_{f}}$ is a mapping given by

$$
f_{R_{f}}: E \rightarrow I^{U},
$$

where

$$
f_{R_{f}}(e)(x)=R_{f}(e, x)(x \in U)
$$

for each $e \in E$, we have

$$
f_{R_{f}}(e)(x)=f(e)(x) \text { for each } x \in U .
$$

Then $f_{R_{f}}(e)=f_{R}(e)$ for each $e \in E$

Thus

$$
f_{E}=\left(f_{R_{f}}\right)_{E}
$$

\section{Theorem 7.5 Let}

$$
\Sigma=\left\{f_{E}: f_{E} \text { is a fuzzy soft set over } U\right\}
$$

and

$$
\Gamma=\{R: R \text { is a fuzzy relation from } E \text { to } U\} .
$$

Then there exists a one-to-one correspondence between $\Sigma$ and $\Gamma$.

Proof. Two mapping $\rho: \Sigma \rightarrow \Gamma$ and $\lambda: \Gamma \rightarrow \Sigma$ are defined as follows:

$$
\begin{gathered}
\rho\left(f_{E}\right)=R_{f}, \text { for any } f_{E} \in \Sigma, \\
\lambda(R)=\left(f_{R}\right)_{E}, \text { for any } R \in \Gamma .
\end{gathered}
$$

By Lemma 7.3,

$$
\rho \circ \lambda=i_{\Gamma},
$$

where $\rho \circ \lambda$ is the composition of $\lambda$ and $\rho$, and $i_{\Gamma}$ is the identity mapping on $\Sigma$.

By Lemma 7.4,

$$
\lambda \circ \rho=i_{\Sigma},
$$

where $\lambda \circ \rho$ is the composition of $\rho$ and $\lambda$, and $i_{\Sigma}$ is the identity mapping on $\Gamma$.

Hence $\rho$ and $\lambda$ are two one-to-one correspondences. This prove that there exists a one-to-one correspondence between $\Sigma$ and $\Gamma$. 
Definition 7.6 $\left({ }^{28}\right)$ Let $U$ be a finite set of objects and let $E$ be a finite set of attributes. The pair $(U, E, V, g)$ is called an information system, if $g$ is an information function specifying the attributesvalue for each object and given by $g: U \times E \rightarrow V$, and $V=\bigcup_{e \in E} V_{e}$ is the value domain of $E$, where $V_{e}=\{g(x, e): x \in U\}$ is the value domain of the attribute e.

Definition 7.7 Let $(U, A, V, g)$ be an information system.

(1) $(U, A, V, g)$ is called a 2-valued information system, if $V=\{0,1\}$.

(2) $(U, A, V, g)$ is called a $[0,1]$-valued information system, if $V=[0,1]$.

Example 7.8 Let $U=\left\{h_{1}, h_{2}, h_{3}, h_{4}, h_{5}, h_{6}\right\}$ be a universe consisting of six patients, and let $A=$ $\left\{a_{1}, a_{2}, a_{3}\right\}$ be a set of attributes where $a_{1}, a_{2}$ and $a_{3}$ represent respectively the attributes " headache", " muscle pain" and "fever".

Now, we consider an information system $(U, A, V, g)$, which describes the " symptoms of patients". For instance, " $g\left(h_{1}, a_{1}\right)=$ yes" means " $h_{1}$ suffers from headache" and its functional value is yes; " $g\left(h_{3}, a_{2}\right)=$ no "means " $h_{3}$ has no muscle pain" and its functional value is no; " $g\left(h_{3}, a_{3}\right)=$ no" means " $h_{3}$ doesn't have a fever" and its functional value is no.

We define

$$
\begin{aligned}
& g\left(h_{1}, a_{1}\right)=\text { yes }, \quad g\left(h_{1}, a_{2}\right)=\text { yes }, \quad g\left(h_{1}, a_{3}\right)=\text { no, } \\
& g\left(h_{2}, a_{1}\right)=y e s, \quad g\left(h_{2}, a_{2}\right)=y e s, \quad g\left(h_{2}, a_{3}\right)=y e s \text {, } \\
& g\left(h_{3}, a_{1}\right)=\text { yes }, \quad g\left(h_{3}, a_{2}\right)=\text { yes, } \quad g\left(h_{3}, a_{3}\right)=\text { no, } \\
& g\left(h_{3}, a_{1}\right)=\text { no, } \quad g\left(h_{3}, a_{2}\right)=\text { yes, } \quad g\left(h_{3}, a_{3}\right)=\text { no, } \\
& g\left(h_{3}, a_{1}\right)=\text { no, } \quad g\left(h_{3}, a_{2}\right)=\text { no, } \quad g\left(h_{3}, a_{3}\right)=\text { yes, } \\
& g\left(h_{3}, a_{1}\right)=\text { no, } \quad g\left(h_{3}, a_{2}\right)=\text { yes }, \quad g\left(h_{3}, a_{3}\right)=\text { yes. }
\end{aligned}
$$

Let $h_{i j}$ be the entries. If $g\left(h_{i}, a_{j}\right)=y e s$, then $h_{i j}=1$; if $g\left(h_{i}, a_{j}\right)=$ no, then $h_{i j}=0 . \quad$ A 2-value information system $(U, A, V, g)$ can be described by the following Table 3.

Table 3. The 2-value information system $(U, A, V, g)$

\begin{tabular}{cccc}
\hline & $a_{1}$ & $a_{2}$ & $a_{3}$ \\
\hline$h_{1}$ & 1 & 1 & 0 \\
$h_{2}$ & 1 & 1 & 1 \\
$h_{3}$ & 1 & 1 & 0 \\
$h_{4}$ & 0 & 1 & 0 \\
$h_{5}$ & 0 & 0 & 1 \\
$h_{6}$ & 0 & 1 & 1 \\
\hline
\end{tabular}

In Table 3,

$$
\begin{gathered}
V_{a_{1}}=\{0,1\}, V_{a_{2}}=\{0,1\}, V_{a_{3}}=\{0,1\} . \\
V=\bigcup_{a \in A} V_{a}=\{0,1\} .
\end{gathered}
$$

Similarly, we may give an example of $[0,1]$ valued information system.

Proposition 7.9 $\left({ }^{30}\right)$ Every soft set may be considered a 2-valued information system.

Proposition 7.10 Every fuzzy soft set may be considered a [0,1]-valued information system.

Proof. Let $\left(f, E^{\prime}\right)$ be a fuzzy soft set over $U^{\prime}$ and let $(U, E,[0,1], g)$ be a $[0,1]$-valued information system. Obviously, the universe $U^{\prime}$ in $\left(f, E^{\prime}\right)$ may be considered the set $U$ of objects in $(U, E,[0,1], g)$, the set $E^{\prime}$ of parameters may be considered the set $E$ of attributes. The information function $g$ is defined by

$$
g(x, e)=f(e)(x)
$$

for each $(e, x) \in E \times U$.

That is, $V=\bigcup_{e \in E} V_{e}$ is the value domain of $E$, where $V_{e}=\{f(e)(x): x \in U\}$ is the value domain of the attribute $e$. Obviously, $V \subseteq[0,1]$.

Therefore, a fuzzy soft set $\left(f, E^{\prime}\right)$ may be considered a $[0,1]$-valued information system $(U, E,[0,1], g)$.

Definition 7.11 Let $S=(f, E)$ be a fuzzy soft set over $U$. Define a function $g_{S}: X \times E \rightarrow V$ by

$$
g_{S}(x, e)=f(e)(x)
$$

for each $(e, x) \in E \times U$. Then $\left(U, E,[0,1], g_{S}\right)$ is called a $[0,1]$-valued information system induced by $S$. We denote it by $I S_{S}$. 
Definition 7.12 Let $I S=(U, E,[0,1], g)$ be a $[0,1]$ valued information system. Define a mapping $f_{I S}$ : $E \rightarrow I^{U}$ by

$$
f_{I S}(e)(x)=g(x, e)
$$

$(x \in U)$ for each $e \in E$, then $\left(f_{I S}\right)_{E}=\left(f_{I S}, E\right)$ is called a fuzzy soft set over $E$ induced by IS. We denote it by $S_{I S}$.

Lemma 7.13 Let $S=(f, E)$ be a fuzzy soft set over $E$, let $I S_{S}=\left(U, E,[0,1], g_{S}\right)$ be a $[0,1]$-valued information system induced by $S$ over $U$ and let $S_{I S_{S}}$ be a fuzzy soft set over $U$ induced by $I S_{S}$. Then $S=S_{I S_{S}}$.

Proof. This is obvious.

Lemma 7.14 Let $I S=(U, E,[0,1], g)$ be a $[0,1]$ valued information system, let $S_{I S}$ be a fuzzy soft set over $U$ induced by IS and let $I S_{S_{I S}}=$ $\left(U, E,[0,1], g_{S_{I S}}\right)$ be a $[0,1]$-valued information system induced by $S_{I S}$. Then $I S=I S_{S_{I S}}$.

Proof. This is obvious.

Theorem 7.15 Let and

$$
\Sigma=\left\{S: S=f_{E} \text { is a fuzzy soft set over } U\right\}
$$

$\Gamma=\{I S: I S=(U, E,[0,1], g)$ is a

$[0,1]$-values informationsystem $\}$.

Then there exists a one-to-one correspondence between $\Sigma$ and $\Gamma$.

Proof. This holds by Lemma 7.13 and 7.14.

\section{Conclusions}

In this paper, we considered a pair of fuzzy soft rough approximations, gave their properties, obtained the structure of fuzzy soft rough sets and the structure of fuzzy topologies induced by fuzzy soft sets, and revealed the fact that every fuzzy topological space is a fuzzy soft approximating space. Moreover, we proved that there exists a one-to-one correspondence between the set of all fuzzy soft sets and the set of all [0,1]-valued information systems, which illustrates that we can research $[0,1]$ information systems by means of fuzzy soft sets. In addition, we also proved that there exists a one-toone correspondence between the set of all fuzzy soft sets and the set of all fuzzy relations from the set of parameters to the initial universe. We may mention that these correspondences are of theoretical significance for the study of soft set theory. We can consider some concrete applications of our proposed notions and theories such as dealing with knowledge acquisition in information systems with fuzzy decisions. In future work, we will study these problems and generalize the results of this paper to t-norms or some non-associative functions such as overlap and grouping functions.

\section{Acknowledgements}

The authors would like to thank the editors and the anonymous reviewers for their valuable comments and suggestions which have helped immensely in improving the quality of this paper. This work is supported by the National Natural Science Foundation of China (11461005, 11061004), the Natural Science Foundation of Guangxi (2014GXNSFAA118001), Guangxi University Science and Technology Research Project (2013ZD020), the Science Research Project of Guangxi University for Nationalities (2012MDZD036), Philosophy and Social Science's Planning Research Topic of Guangxi (13FJL004), the Science Research Project 2013 of the ChinaASEAN Study Center (Guangxi Science Experiment Center) of Guangxi University for Nationalities (KT201310, KT201315) and Key Laboratory of Optimization Control and Engineering Calculation in Department of Guangxi Education.

\section{References}

References are to be listed in the order cited in the text. Use the style shown in the following examples. For journal names, use the standard abbreviations. Typeset references in 9 pt Times Roman.

1. M.I.Ali, "A note on soft sets, rough soft sets and fuzzy soft sets," Appl. Soft Comput., 11, 3329-3332 (2011).

2. H.Aktas, N.Cağman, "Soft sets and soft groups," Inform. Sci., 177, 2726-2735 (2007).

3. C.Chang, "Fuzzy topological spaces," J. Math. Anal. Appl., 24, 182-190 (1968).

4. A.Császár, "Generalized topology, generalized continuity,” Acta Math. Hungar., 96, 351-357 (2002). 
5. A.Császár, "Generalized open sets," Acta Math. Hungar., 75, 65-87 (1997).

6. D.Dubois, H.Prade, "Rough fuzzy sets and fuzzy rough sets," Int. J. General Systems, 17, 191-209 (1990).

7. F.Feng, C.Li, B.Davvaz, M.I.Ali, "Soft sets combined with fuzzy sets and rough sets: a tentative approach," Soft Comput., 14, 899-911 (2010).

8. F.Feng, X.Liu, V.Leoreanu-Fotea, Y.B.Jun, "Soft sets and soft rough sets," Inform. Sci., 181, 1125-1137 (2011).

9. F.Feng, Y.B.Jun, X.Liu, L.Li, "An adjustable approach to fuzzy soft set based decision making," J. Comput. Appl. Math., 234, 10-20 (2010).

10. X.Ge, Z.Li, Y.Ge, "Topological spaces and soft sets," J. Comput. Anal. Appl., 13, 881-885 (2011).

11. Y.Jiang, Y.Tang, Q.Chen, J.Wang, S.Tang, "Extending soft sets with description logics," Comput. Math. Appl., 59, 2087-2096 (2010).

12. Y.Jun, "Soft BCK/BCI-algebras," Comput. Math. Appl., 56, 1408-1413 (2008).

13. Y.B.Jun, K.J.Lee, C.H.Park, "Fuzzy soft set theory applied to BCK/BCI-algebras," Comput. Math. Appl., 59, 3180-3192 (2010).

14. Z.Li, D.Zheng, J.Hao, "L-fuzzy soft sets based on complete Boolean lattices," Comput. Math. Appl., 64, 2558-2574 (2012).

15. Y.Liu, M.Luo, "Fuzzy Topology", World Scientific Publishing, Singapore, 1998.

16. P.K.Maji, R.Biswas, A.R.Roy, "Soft set theory," Comput. Math. Appl., 45, 555-562 (2003).

17. P.K.Maji, R.Biswas, A.R.Roy, "Fuzzy soft sets," J. Fuzzy Math., 9, 589-602 (2001).

18. D.Molodtsov, "Soft set theory-first result," Comput. Math. Appl., 37, 19-31 (1999).

19. D.Meng, X.Zhang, K.Qin, "Soft rough fuzzy sets and soft fuzzy rough sets," Comput. Math. Appl., 62, 46354645 (2011).

20. Z.Pawlak, "Rough sets: Theoretical sspects of reasoning about data", Kluwer Academic Publishers,
Boston, 1991.

21. B.Pu, Y.Liu, "Fuzzy Topology I. Neighborhood structure of a fuzzy point and Moore-Smith convergence," J. Math. Anal. Appl., 76, 571-599 (1980).

22. Z.Pei, D.Pei, L.Zheng, "Topology vs generalized rough sets," Inter. J. Approx. Reason., 52, 231-239 (2001).

23. K.Qin, Z.Pei, "On the topological properties of fuzzy rough sets," Fuzzy Sets and Systems, 151, 601-613 (2005).

24. A.R.Roy, P.K.Maji, "A fuzzy soft set theoretic approach to decision making problems," J. Comput. Appl. Math., 2003, 412-418 (2007).

25. B.Tanay, M.B.Kandemir, "Topological structure of fuzzy soft sets," Comput. Math. Appl., 61, 2952-2957 (2011).

26. X.Yang, T.Lin, J.Yang, Y.Li, D.Yu, "Combination of interval-valued fuzzy set and soft set," Comput. Math. Appl., 58, 521-527 (2009).

27. L.A.Zadeh, "Fuzzy sets," Inform. Control, 8, 338-353 (1965).

28. W.Zhang, W.Wu, J.Liang, D.Li, "Rough sets theorey and methods", Chinese Scientific Publishers, Beijing, 2001.

29. X.Zhang, J.Dai, Y.Yu, "On the union and intersection operations of rough sets based on various approximation spaces," Inform. Sci., 292, 214-229 (2015).

30. Y.Zou, Z.Xiao, "Data analysis approaches of soft sets under incomplete information," Knowledge-Based Systems, 21, 941-945 (2008).

31. L.Yang, L.Xu, "Topological properties of generalized approximation spaces," Inform. Sci., 181, 3570-3580 (2011).

32. M.I.Ali, F.Feng, X.Liu, W.K.Min, M.Shabir, "On some new operations in soft set theory," Comput. Math. Appl., 57, 1547-1553 (2009).

33. M.Shabir, M.Naz, "On soft topological spaces," Comput. Math. Appl., 61, 1786-1799 (2011). Information Sciences 292 (2015) 